\title{
Agustín de Pedrayes en su contexto. Matemática sublime y aplicada
}

\author{
José Manuel Álvarez Pérez \\ IES. Jerónimo González, Langreo
}

CES.XVIII, núm. 24 (2014), págs. 7-47. 


\section{RESUMEN}

El 26 de febrero de 2015 se cumple el segundo centenario de la muerte de Agustín Bernardo de Pedrayes y Foyo, matemático ilustrado del siglo xvIII. Este artículo es un breve bosquejo de su biografía y del estado de la ciencia matemática en aquella España. Además anunciamos la transcripción de sus obras manuscritas más importantes, en espera de una edición crítica, Nuevo y Universal Método de cuadraturas determinadas y el Programa-Problema presentado a las Academias de París, Berlín y San Petesburgo, como ejemplo de sus aportaciones a la matemática sublime del análisis diferencial.

Con este trabajo se pretende demostrar que Agustín Bernardo de Pedrayes y Foyo tiene una importancia capital en la Historia de la Ciencia, en lo referente a la investigación matemática, superior a la que se le ha dado.

Palabras clave

Matemática, Ilustración, Agustín de Pedrayes y Foyo, Historia de la ciencia.

\section{ABSTRACT}

On February 26, 2015 the second anniversary of the death of Agustín Bernardo de Pedrayes y Foyo illustrated mathematician of the XVIII century. This article is a brief outline of his biography and the situation of mathematical science in the Spain. Also the author announce the transcription of its most important manuscript works, at this time waiting for a critical edition, New and Universal Method of certain quadratures and the Program-Problem presented to the Academy of Paris, Berlin and St. Petersburg, as an example of his contributions to mathematics sublime and differential analysis.

This paper aims to demonstrate that Agustín Bernardo de Pedrayes y Foyo has a big importance in the History of Sciences, spcially in the mathematical research, higher than it has been given.

KEY WORDS

Mathematics, Illustration, and Foyo Pedrayes Augustine, History of science.

Recibido: 15 de septiembre de 2014. Aceptado: 11 de noviembre de 2014. 


\section{Introducción}

El nombre de Agustín de Pedrayes ha sido conocido y apreciado por las instituciones más insignes del mundo científico de su época, en aquellas naciones europeas donde se honraba la ciencia matemática y a los hombres que la hicieron posible. Pero su nombre no ha recibido todavía el homenaje, al menos en España, de que es acreedor, bien por ignorancia, bien por desconocimiento de su obra, bien por ideológicos prejuicios. Es por tanto, de obligado cumplimiento la necesidad de un gran reconocimiento que debe dársele por ilustre y por justicia. El mismo homenaje, valoración y respeto a su aportación a la matemática, que le brindaron los más ilustres académicos, científicos y matemáticos de toda Europa a fines del siglo XVIII y primera mitad del siglo XIX, debe ser expresado hoy con total contundencia. Durante cerca de cuarenta y cinco años, entre 1769 y su muerte en 1815, Pedrayes consagró su vida a la enseñanza, al estudio y a la creación de la matemática sublime y también de la aplicada, entregando vida y salud a los asuntos más importantes que puedan interesar al hombre pensador y científico.

Acerca de la obra de Pedrayes se han hecho en España estudios y homenajes desde finales del siglo XIX y a lo largo del siglo XX. En 1881, se publicó en la Ilustración Gallega y Asturiana, número 3, una breve recensión sobre el matemático Pedrayes: «Excmo. Señor D. Agustín Pedrayes y Foyo» de Eugenio Ruidíaz y Caravia. Y el 26 de febrero de 1925, el mismo Eugenio Ruidíaz y Caravia publica una breve biografía. El 25 de marzo de 1929, Julio Martínez Hombre da a la luz un trabajo sobre la vida de Pedrayes, titulado Un sabio asturiano Agustín de Pedrayes ${ }^{1}$. En 1951 Javier Rubio Vidal publica Un matemático asturiano casi olvidado, Agustín de Pedrayes ${ }^{2}$, hasta la fecha el trabajo más documentado y completo. Ramón Crespo Pereira, en 1952, publica Agustín de Pedrayes $^{3}$. Y en 1953, «Agustín de Pedrayes, el matemático español más ilustre del siglo XVIII», Cuadernos Hispanoamericanos, 45 (1953), págs. 319-330. El 11

\footnotetext{
1 Julio Martínez Hombre, «Un sabio asturiano. El matemático Agustín de Pedrayes». Revista Asturias (número extraordinario), 25 de marzo de 1929, págs. 57-59.

2 Javier Rubio Vidal, Un matemático asturiano casi olvidado, Agustín de Pedrayes. Discurso de recepción en IDEA, 20 de diciembre de 1950; contestación de Ignacio Patac Pérez, Oviedo, IDEA, 1951.

3 Ramón Crespo Pereira, «Agustín de Pedrayes», Gaceta Matemática, IV (1952), págs. 3-8.
} 
de noviembre de 2008 tiene lugar una conferencia de Pablo Suárez, en el «Día de las ciencias asturianas». El mismo Pablo Suárez García publica en bable un trabajo más amplio, titulado Un matemáticu asturianu del sieglu XVIII: Agustín Bernardo de Pedrayes y Foyo (1744-1815)4. Los citados trabajos de Rubio Vidal y de Pablo Suárez García reunieron toda la documentación de que disponían hasta la fecha de su publicación.

Todas estas publicaciones fueron trabajos en revistas, periódicos y publicaciones divulgativas, y todos valoraron la importancia de Pedrayes en cuanto enseñante como matemático y como representante en la Oficina de Pesas y Medidas de París. Pero no existe un estudio riguroso de las aportaciones de Pedrayes a la matemática sublime, es decir la matemática diferencial e integral, a través de los manuscritos del propio Pedrayes y de otros documentos de los que disponemos hoy, publicados por los matemáticos más insignes de la época de Pedrayes.

Sus aportaciones a las cuadraturas, la aplicación de las ecuaciones diferenciales al cálculo de superficies limitadas por curvas, cincuenta años antes de que Riemann definiera su famosa integral de superficie; el conocimiento y desarrollo del cálculo diferencial e integral tan reciente en su época; la inmensa aportación a la definición del metro como base del nuevo Sistema de Pesas y Medidas, así como sus conocimientos sobre los planes para la enseñanza de la matemática, fueron algunos de los hitos de su tarea como profesor e investigador. Y como ingeniero práctico de la matemática aplicada, la creación de dos barómetros y del comparador. En definitiva: todos sus esfuerzos fueron culminados por aportaciones de gran valor, de manera premonitoria a las grandes aportaciones de la matemática en el siglo XIX.

Desde niño demostró sus cualidades para los estudios de la matemática y de las lenguas. Su primera orientación, por influencia de su tío, fue hacia la teología, el derecho canónico y la filosofía escolástica. Pronto, aquel niño, que soñaría un día con echarse a la mar y ser un importante bucanero, como muchos otros niños de su Lastres natal, brilló durante sus estudios en Santiago de Compostela obteniendo bachillerato y licenciatura. Se supone que por influencia del benedictino tío suyo que, al igual que otros benedictinos como Feijoo y Sarmiento, tuvo especial interés por la matemática y la ciencia en general, Agustín Bernardo de Pedrayes comienza a estudiar matemáticas.

Se titula en la Universidad de Santiago en Filosofía, Teología y Leyes obteniendo el graduado en 1762 y en 1769 fue nombrado Maestro de Matemáticas

4 Pablo Suarez García, Un matemáticu asturianu del sieglu XVIII: Agustín Bernardo de Pedrayes y Foyo (1744-1815), Oviedo, Conseyería de Cultura y Turismu, 2008. 


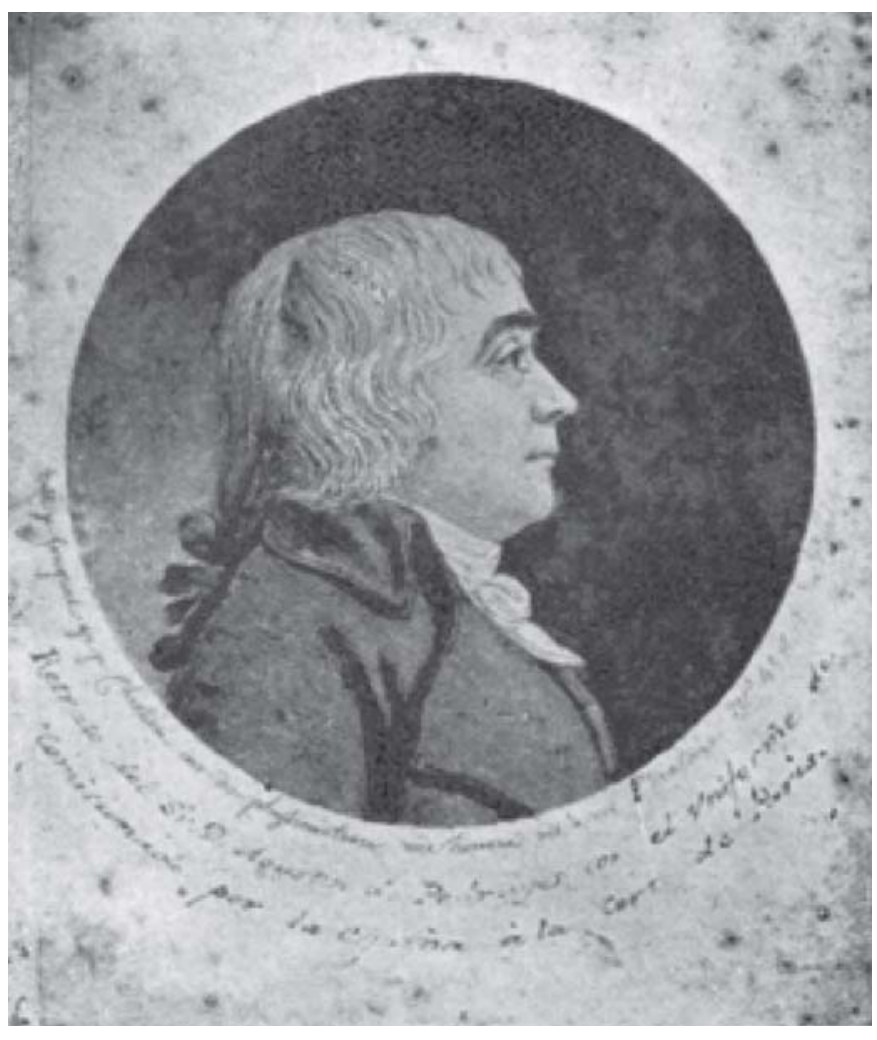

Leyenda que figura en el retrato de la familia: Retrato de D. Agustín de Pedrayes con el uniforme de comisionado por la España a la Corte de París

de la Real Casa de Caballeros Pajes de su Majestad, lugar donde enseñó hasta 1786. Posteriormente continuó Pedrayes sus explicaciones en el Seminario de Nobles, hasta el año 1791.

En un espacio de tiempo corto, obtuvo pleno conocimiento de la más alta y abstracta matemática. Desde entonces se dejó caer en la seducción y las redes de esa ciencia a la que entregó sin reservas todo su genio. No está claro el motivo por el que es llamado a Madrid para dirigir la enseñanza de la Matemática en el Colegio de Pajes. Se ha querido buscar el origen de dicho destino académico a través de alguna recomendación. La realidad fue que la capacidad de Agustín de Pedrayes no parece que necesitara recomendaciones. Fue valorado por sus conocimientos profundos de la matemática en aquellos momentos y nadie podía en la Corte española probar más capacidad que él para ampliar los dominios de dicha ciencia. Sea como fuere, Pedrayes se encuentra en 1769 enseñando en el colegio de los jesuitas, actividad que mantendrá hasta su retiro por enfermedad en 1791.

A los pocos años de su estancia en Madrid, exactamente en 1777, Pedrayes deslumbra a los más importantes matemáticos europeos con la presentación del Nuevo y Universal Método de cuadraturas determinadas (MS. 8668 Biblioteca Nacional de España). El manuscrito de dicho trabajo fue olvidado por los pocos 
biógrafos que tuvo Agustín de Pedrayes. Al estudiar dicho manuscrito, ignorado hasta ahora, se entiende la potencialidad del mismo. En él, ya plantea una nueva forma de resolver cuadraturas con el instrumento de la matemática sublime, tal como se denominaba por entonces el análisis diferencial; su genio nos trajo una nueva forma de iniciar el estudio analítico de la circunferencia partiendo del Teorema de Thales, cuestión que bien podría estar en los más importantes manuales de Historia de la Matemática. Había resuelto un problema fundamental de la matemática después que Descartes y Fermat iniciaran la geometría analítica y de que Newton y Leibnitz hicieran lo propio con el análisis diferencial. La sublime ciencia era de su posesión y podía abarcarla y perfeccionarla en toda su extensión.

Diecinueve años después y tras una larga enfermedad, presentará un problema similar en forma de reto a la comunidad matemática internacional, concentrada en las Academias de Ciencias de París, Berlín y San Petesburgo, sin que ninguno de los importantes matemáticos de la época pudiera resolverlo. En dicho trabajo, titulado Opúsculo I (Programa con nuevo método para resolver el Problema de hallar una ecuación diferencial correspondiente a otra integral), Pedrayes plantea resolver una ecuación diferencial de dieciséis términos que, en opinión de Gauss ${ }^{5}$, podría tratarse de una forma de resolver integrales elípticas. Schumacher escribe a Gauss (Altona, 2 de abril de 1808):

Hace unos 10 años, el español Pedrayes propuso a todos los matemáticos una ecuación diferencial para integrar, que está publicada en Göttingische gelehrte Anzeigen, unter der Aufsicht der Gesellschaft der Wissenschaften (Anuncios académicos bajo la supervisión de la Sociedad de Ciencias) y en el archivo de Hindenburg. Pfaff tiene integrada la ecuación diferencial sólo para un caso particular (por lo que recuerdo, solo pone dos variables de la misma), por el contrario, que yo sepa, ya nada parece tan raro como que ya, en vida suya (de usted), de Laplace y de Lagrange, un extranjero pudiera desafiar a tales hombres en vano. Por supuesto, él dice que nuevos métodos, métodos que él ha diseñado, serían necesarios, ¿̨pero no podría haberlos alcanzado indirectamente, lo que sería una manera más corta para llegar a la solución? ¿Y lo que él ha inventado, no podría reinventarse?

Cinco meses después, en carta de Friedrich Gauss a Schumacher el 17 de septiembre de 1808, contesta y dice:

5 Carl Friedrich Gauss; Heinrich Christian F. Schumacher, Briefwechsel Zwischen C.F. Gauss und H.C. Schumacher, (ed. C.A.F. Peters), Altona, [s.n.], 1860. Cartas 1 y 2. 
Ahora sabemos cómo lidiar con funciones circulares y logarítmicas, como con relación uno a uno, aunque la gloriosa mina de oro que ocultan las funciones más elevadas aún es casi Tierra Incógnita. He trabajado mucho sobre ello anteriormente, y un día crearé un gran trabajo sobre ello, sobre lo que ya he dado una muestra en mis Disquisitiones Arithmeticae, pp. 593. Uno cae en el asombro ante la profusa riqueza de las nuevas y altamente interesantes verdades y relaciones tales como las funciones presentadas (donde, entre otras, están también aquellas asociadas realmente con la rectificación de la elipse y la hipérbola). Bien podría ser que la respuesta al problema de Pedrayes pudiese hallarse justamente en estos estudios, siempre y cuando permitan una solución que realmente tenga valor: sólo si viese aún más claro que el problema completo pudiese conducir a algo, como no es el caso hasta ahora, pero de momento debo abstraerme de este estudio, porque sólo entonces me sumergiría de nuevo en esta profunda materia, cuando sea capaz de pensar en el desarrollo de esa gran obra.

Aunque ha llegado hasta nosotros la solución que ofreció Pedrayes en 1805, no hemos podido recuperar el Opúsculo II en el que ampliaba la solución a dicho problema, por haber desaparecido en la quema del Observatorio de Madrid por las tropas napoleónicas, durante la Guerra de la Independencia.

Agustín de Pedrayes dedicó toda su vida al estudio de estos y otros problemas matemáticos, con tal constancia que sus aportaciones fueron consideradas con interés más allá de nuestras fronteras. Quiso añadir su aportación al problema histórico de las cuadraturas, problema que desde la antigua Grecia, en particular desde Arquímedes y Apolonio, estuvo en el centro del pensamiento matemático más refinado. El tiempo fue aclarando el olvido de los mediocres que siempre quisieron ver con mayor interés los éxitos de otras latitudes, sin conocer las importantes aportaciones de nuestros sabios del XVIII. Mas la mediocridad puede retrasar el conocimiento de las grandes obras, pero no puede enterrarlas.

Y es así como el nombre de Pedrayes perdurará a todas las épocas junto a los grandes matemáticos de la segunda mitad del siglo XVIII y principios del XIX. Las ciencias matemáticas han tenido en la Europa del siglo XVIII a grandes genios como Euler, Lagrange o Fourier. Y también han tenido a Pedrayes. Los escritos de este gran matemático no deberán permanecer por más tiempo olvidados y la comunidad deberá ponerse a estudiarlos críticamente y a valorarlos. Lo mismo que Lagrange había inventado y engrandecido buena parte del cálculo, Agustín de Pedrayes hizo lo propio con las cuadraturas y los inicios de la geometría diferencial.

La principal y más significativa peculiaridad de Agustín de Pedrayes no era otra que su extrema sencillez, su constancia en el trabajo y un desinteresado protagonismo. Dedicó toda su vida al estudio incansable de la matemática 
y su enseñanza. Hasta el punto que a mitad de su carrera hubo de retirarse a su pueblo natal gravemente enfermo. En un mundo encorsetado por las trabas inquisitoriales y religiosas, su pensamiento simple, pero elevado y sin fronteras, abarcó lo más novedoso y avanzado de la matemática de su tiempo. Dominador de las lenguas de nuestro entorno geográfico, leía y estudiaba directamente a los creadores matemáticos de toda Europa en sus lenguas y sin epígonos; los interpretaba y los desarrollaba proyectando su ciencia a un estadio superior. $\mathrm{Su}$ constancia vencía obstáculos, renovaba conceptos y aportaba las formas más ingeniosas y diversas para su resolución.

De esa manera presentó el problema de las cuadraturas en 1777. En dicho manuscrito, comienza el estudio de las cuadraturas así:

Después de la Invención de los Cálculos diferencial e integral se han hecho en las Ciencias Mathemáticas puras y mixtas, progresos tan rápidos que no pueden dejar de admirarse; manifestándose por este hecho quanto un methodo descubierto en una Ciencia qual es la Geometría puede influir en el adelantamiento de todas las demás.

Persuadidos de esta verdad los más célebres mathemáticos se han dedicado con el mayor esmero, especialmente en este siglo, a perfeccionar uno y otro Cálculo, hallando muchos méthodos, tanto particulares como generales muy ingeniosos y útiles; entre los que son ciertamente recomendables el méthodo de las Variaciones, el de distinguir las diferenciales que no incluyen condición imposible, y el de señalar a qualquiera de estas, propuesta su integral.

Pero entre las mismas integrales hay muchas que aunque son reales no pueden expresarse si no por series de infinitos términos, y a pesar de los grandes esfuerzos que hasta ahora se han hecho, los methodos sabidos no alcanzan a más que a distinguir aquellas que pueden reducirse a ciertas fórmulas conocidas.

En las quadraturas de las curvas es en donde particularmente se experimenta que las expresiones de las diferenciales de las areas son regularmente incompletas, y les falta algún término para que les correspondan integrales expresadas en términos finitos; lo que hace con razón se miren como imposibles sus expresiones por fórmulas finitas.

De ahí han inferido muchos que es imposible la quadratura exacta de aquellas curvas en las que las diferenciales de las áreas no tienen integrales completas y que por consiguiente podrán solamente expresarse por series de infinitos términos $^{6}$; del mismo modo que no siendo posible la expresión de un radical por ejemplo

${ }^{6}$ Se refiere a la posibilidad de resolver integrales definidas para hallar áreas bajo curvas sin utilizar las sumas infinitas o la integración numérica. 
por números finitos se podrá solamente tener aproximada por una serie infinita; y como saben todos quanto a este intento se ha trabajado en vano desde la antigüedad hasta ahora, se tiene ya como una especie de locura, o manía mathemática la ocupación en tales investigaciones? ${ }^{7}$.

Este problema que había sido preocupación desde Eudoxo, Arquímedes y Apolonio, en la antigua Grecia, y que sería resuelto a mediados del siglo XIX, por Lindemann en 1882, demostrando la imposibilidad de cuadrar el círculo con regla y compás, y con Riemann hallando áreas bajo curvas, por medio de la integral que lleva su nombre, era uno de los problemas fundamentales que podía proponerse la inteligencia humana.

Pedrayes no llegó a comprender las integrales de superficie, pero con su resolución del problema planteado en 1799 a las Academias de París, Berlín y San Petesburgo, hizo una extraordinaria aportación para la solución encontrada a mediados del siglo XIX, en particular para la obtención de los coeficientes en una ecuación diferencial. En su obra Nuevo y Universal Método de cuadraturas determinadas, presenta una de las aportaciones más imaginativas de aquella primera época de creador: la obtención de la ecuación cartesiana del círculo a partir del Teorema de Thales. En 1796 presentó el Problema-Programa, que dos años después sería objeto de certamen a concurso, para las Academias de ciencias de París, Berlín y San Petesburgo. Presentó la solución ${ }^{8}$ en 1805 en forma de Opúsculo $I$.

Sus importantes descubrimientos ayudaron a perfeccionar los aspectos más importantes del análisis infinitesimal. Y también tuvieron una consecuencia inmediata en la medición del metro y su colaboración en la medición del arco de cuadrante de meridiano entre Barcelona y Dunkerque.

Todo lo referente a la geometría y a la medida, tuvo en él gran dedicación y un importante objeto de estudio. Sometió al cálculo más detallado la medida de superficies diversas, en particular el círculo, la parábola y las cónicas en general.

Del conjunto de sus investigaciones debemos subrayar la medición de cuadraturas con aplicación del cálculo diferencial a la búsqueda de áreas limitadas por curvas (en particular de cónicas), tal como él nos refiere. Pero no debemos olvidar su sentido de la didáctica de la matemática.

\footnotetext{
7 Agustín Pedrayes Y Foyo, Nuevo y Universal Método de cuadraturas determinadas (Ms. 8668, Biblioteca Nacional de España, 1777). <http://bdh.bne.es/bnesearch/detalle/bdh0000164624>

8 Solución del Problema Propuesto el año de 1797 [Agustín Pedrayes]; Dado a luz por una Asociación Literaria. Madrid, en la Imprenta de la Administración del Real Arbitrio de Beneficencia, 1805. <http://bvpb. mcu.es/es/catalogo_imagenes/grupo.cmd?path=387>
} 
Fue comisionado por el gobierno de Carlos IV para participar junto con Gabriel de Ciscar y Ciscar 9 , en el Congreso de Pesas y Medidas de París, en 1799. En París se ganó la admiración y el aprecio de los científicos europeos más importantes de la época, siendo conocido como «el sabio español». Y según Menéndez y Pelayo publica un Manual de matemáticas del que no tenemos referencia.

Sus aportaciones han sido variadas y diversas como el «comparador»o medidor de longitudes ${ }^{10}$. Buscaba la perfección en todo lo que estudiaba; siempre buscaba el mayor rigor en la creación y desarrollo de sus instrumentos de medida. Y también la perfección de los instrumentos útiles. Además del comparador que construye con la colaboración del francés Étienne Lenoir, también construyó dos barómetros.

Tenía Pedrayes una capacidad de trabajo superior para ser lo más meticuloso posible en la exposición de sus trabajos. Con una vida moderada, de gran sencillez en las costumbres y un apego inquebrantable a los intereses generales, al bien común y a la causa de la verdad y la libertad, no es extraño que experimentara gran empatía con Jovellanos. Lo mismo podía escribir el famoso problema de las cuadraturas que elaborar el programa de estudios matemáticos del Colegio de Nobles, que colaborar en la elaboración de los programas de ciencias para el Instituto que Jovellanos proyectaba en Gijón. Lo mismo participaba activamente y con admiración del resto de comisionados en la Oficina de Pesas y Medidas que elaboraba el aparato de medida, el «comparador». Lo mismo defendía la libertad en las Cortes de Cádiz y firmaba la disolución de la Inquisición, que proponía al resto de la comunidad científica europea el Problema planteado a las Academias de París, Berlín y San Petesburgo, en 1796.

Todo aquello en lo que participó recibió el acierto de Pedrayes, con toda humildad, pero también con toda eficacia. De suerte que ninguno de sus con-

9 Ciscar fue a esa Comisión por ser un militar de reconocido prestigio, e ilustrado liberal con buenas relaciones en la estructura estatal. Pedrayes le acompaña por su condición de matemático teórico, cuyo prestigio era conocido entre los más importantes matemáticos europeos.

10 La mayor parte de los instrumentos científicos inventados durante el siglo XVIII se encuentran en la Biblioteca y Museo Arqueológico Nacionales, en el Observatorio Fabra, en la Mentora Alsina y el Museo Naval. En la descripción del instrumento llamado «Comparador» que hace Rubio Vidal, en su obra: Javier Rubio Vidal, Un matemático asturiano casi olvidado, Agustín de Pedrayes. Discurso de recepción en IDEA, 20 de diciembre de 1950; contestación de Ignacio Patac Pérez, Oviedo, IDEA, 1951, se dice: «Con este instrumento se pueden medir todas las longitudes desde una línea hasta 37 pulgadas de la toesa de Francia con la diferencia de una milésima de línea, lo que no puede conseguirse con ningún otro instrumento conocido hasta aquí que tenga la misma exactitud. D. Agustín de Pedrayes le hizo construir en París de orden de S. M. con el fin de que puedan compararse las diferentes varas de España entre sí y con el metro...». 
temporáneos europeos dejó de reconocer su gran valía. Con sólida creatividad resolvió los problemas más importantes y ciertamente más difíciles de todos los considerados hasta entonces por la comunidad matemática.

No podemos afirmar que Pedrayes haya creado una ciencia nueva como lo hicieron en su época Euclides, Descartes o Newton. Ni que haya aportado trabajos de gran extensión con principios originales como Leibnitz o Euler. Ni que haya sido el primero en resolver plenamente el importante problema de las cuadraturas. Pero sí que ha perfeccionado todo aquello de la matemática sublime y aplicada que tocó; que profundizó y extendió los límites de lo que entonces se conocía; y que llevó a resolver lo que hasta entonces se consideraba irresoluble. Y que ha sentado bases y premisas para que otros «caminaran a hombros de gigantes».

Ese fue el carácter que Pedrayes imprimió a todos sus trabajos e investigaciones. Cuestiones desconocidas en aquel momento cuyos conocimientos se incorporaron más adelante al campo del conocimiento humano como feliz suplemento de las imperfecciones de nuestros pensamientos. Esta vena inquieta y creadora fluía de Agustín de Pedrayes como rasgo característico de su personalidad.

Las teorías más abstractas alcanzan en él una belleza que resulta intrínsecamente propia. Los nuevos conceptos, en su época mucho más actuales, como su relación con las aplicaciones prácticas de la matemática sublime, pasaron a motivar y satisfacer su espíritu.

No es ésta una biografía al uso de épocas anteriores. El Pedrayes del que hablamos es más bien un estilo clásico de literatura biográfica; un personaje ilustrado, y científicamente laico.

Mas como todo tratamiento de nuestro biografiado, requiere apoyarse en los hechos de su vida, en los títulos y contenidos de su obra, en la ciencia a la que dedicó su tiempo, con objetiva documentación de los temas investigados, y en los comentarios pertinentes. Es decir, ocuparse de su obra reflexivamente, y no seguir las opiniones de algunos epígonos que por boca de terceros hablan e interpretan a nuestro personaje.

Pedrayes en el siglo XVIII

Cuando nos adentramos en el siglo XVIII, en la etapa de las luces y de la Ilustración, en particular en la historia de su ciencia matemática, nos asombra el progreso llevado a cabo. Mas, cuanto mayor es su estudio y conocimiento, comprobamos que no hubo período de la historia universal en el que hubiera 
tanta difusión e intercambio de ideas, teorías y manifestaciones científicas como en la cuna europea de esa época. El espectáculo del mundo conmovido por la profusión, desarrollo y aplicación de la ciencia trajo un nuevo cambio en las sociedades europeas que la historia se encargó de colocar en el correspondiente lugar e importancia. De hecho, la revolución industrial fue el resultado de tal revolución científica y, a través de ella, de los cambios económicos, sociales, culturales y políticos que se obraron.

En nombre de la Historia, nadie se atrevería hoy a desarrollar un estudio histórico del siglo XVIII sin tener en cuenta sus artes, sus oficios y sus letras; y todo ello, aunque en nuestro país sigan apareciendo manuales y enciclopedias de rancia raigambre, para los cuales la historia de la ciencia es sólo un apéndice.

Nadie podrá negar que los estudios de cartografía, geodesia, astronomía, física, química, medicina, y un largo etc., fueron una necesidad de las épocas de la Edad Moderna y un enorme progreso para la náutica, la navegación, la métrica; para paliar epidemias; para desarrollar la industria textil... y para tantas otras facetas de las economías del XVI, XVII, XVIII y XIX, que cambiaron nuestras sociedades.

Las creaciones matemáticas de aquella época tuvieron mucho que ver con éstas y otras muchas aplicaciones. A consecuencia de los grandes acontecimientos de aquel siglo y de los beneficios que permitieron lograr, se pudo construir una sociedad de mayor progreso. En consecuencia, podemos afirmar en una primera reflexión, que el desarrollo de la matemática del siglo XVIII estuvo determinado por las necesidades del desarrollo social de la época.

Que la ciencia influyó en el desarrollo social, e influye, nadie es capaz, en rigor, de negarlo hoy. Y que por tanto no se puede estudiar la historia política sin conocer lo que hicieron los hombres de ciencia. Y viceversa.

Por otro lado, en las monarquías absolutas del siglo XVIII se gestó la suplantación de la sociedad gremial, pasando de la sociedad corporativa y estamental del viejo régimen a otra sociedad de clases con características bien diferentes. Las nuevas técnicas basadas en los desarrollos científicos, imponían nuevas estructuras sociales. En consecuencia, la nueva industria y la novedosa tecnología, empezando por el maquinismo, no necesitaban de los estamentos del clero y la nobleza. Bajo el yugo de los monarcas absolutos se estaba gestando una nueva estructura social propiciada por los avances científicos y tecnológicos. En el mundo ilustrado europeo era vivísima la empatía y colaboración con los hombres de ciencia en las Academias, Colegios e instituciones militares. Surgían fábricas y caminos de hierro. Los nuevos burgueses propiciaban los nuevos desarrollos tecnológicos y potenciaban la ciencia. 
Consecuentemente, los avances en el estudio de la ciencia y en particular de la matemática, fueron y son determinantes para los cambios estructurales que tienen lugar en cada época.

Por eso hoy resulta extraño comprobar cómo la historia de la ciencia sigue siendo la gran postergada en España, aunque haya aumentado el flujo de publicaciones en las últimas décadas. Según Juan Vernet Ginés en su Historia de la ciencia española, Madrid, Instituto de España, Cátedra Alfonso X el Sabio, 1975:

En el Antiguo Continente se profesan enseñanzas de Historia de la Ciencia en la Universidad de Barcelona (Historia de la Ciencia Árabe entendida en sentido amplio). Sin embargo, esta materia, como otras que se profesaban en las universidades españolas (Historia de la Matemática —Madrid—, Historia de las Ciencias Naturales —Madrid y Barcelona—), está llamada a desaparecer cuando entren en vigor los nuevos planes de estudio. Existen, eso sí, distintas instituciones agrupadas en torno a las moras o agregaciones de Historia de la Medicina y de la Farmacia y del Instituto de Historia de la Medicina y de las Ciencias Naturales «Arnaldo de Vilanova» del CSIC. La neta regresión de la Historia de la Ciencia en España contrasta con la expansión que experimenta en el resto de Europa.

La importancia de Pedrayes estriba en que en este medio introduce un espíritu científico laico, ajeno a cualquier prejuicio religioso y sus ideas se relacionan con las ideas ilustradas predominantes en los ambientes científicos europeos. Pedrayes quería introducir en la inquietud de la sociedad española y de sus gobernantes en la segunda mitad del siglo XVIII, las orientaciones de los inicios industriales y tecnológicos de la Europa occidental. En particular, su mayor interés se centraba en lo que se conoce como la matemática aplicada y la formación científica de las élites dirigentes, fueran militares o civiles.

En los regímenes absolutos de Europa, el pueblo no gozaba de libertad y aspiraba a la libertad plena. En aquellos lugares, como en Francia, en los que las ideas ilustradas habían penetrado más, la libertad era mayor. De hecho, en ninguna parte se habían propagado más las ideas que Rousseau había expresado claramente de libertad, solidaridad y fraternidad, como en Francia.

En España, esas ideas proyectadas a los estudios universitarios y científicos, chocaban con la tradición más rancia de una semiteocracia dirigida por los más reaccionarios de la sociedad.

El estado de letargo y crisis de la universidad española, muy considerable ya en el siglo XVI, quiso superarse con la creación de instituciones paralelas, 
como la Academia de Matemáticas ${ }^{11}$ creada en tiempos de Felipe II y el Colegio Imperial ${ }^{12}$ en Madrid. Estas instituciones, aunque dirigiéndose a niveles muy distintos, buscaron paliar la ineficacia de la universidad a causa de su aristotelismo y escolástica. Pero las instituciones más beneméritas a este respecto durante los siglos XVII y XVIII resultaron ser las academias, cuyo origen en Europa hay que buscar en las tertulias científicas que celebraban los sabios en Italia. En Europa las Universidades y las Academias serán centros difusores de ese espíritu ilustrado. La Royal Society, en Londres (1662); la Académie Royale des Sciences, en París (1666); la Academia Imperialis Scientiarum, en San Petesburgo (1724); la American Philosophical Society, en Filadelfia (1743); la Académie Royales des Sciences et Belles Lettres de Berlín (1744), son algunas de las academias y sociedades creadas en los siglos XVII y XVIII. Por esta razón, nuestro Agustín de Pedrayes planteará su Programa con el Problema, en 1797 a las Academias de Ciencias de París, Berlín y San Petesburgo, y no a las Universidades.

El siglo XVIII supuso un cambio en el campo de las ideas y de la ciencia, en Europa. Un siglo de clara influencia francesa, lugar y cuna de las ideas de la Ilustración. A diferencia de Francia, Inglaterra, Escocia y Alemania septentrional, en España en la primera mitad del siglo XVIII hubo poco brillo de las luces, salvo reducidos núcleos de intelectuales; de hecho hasta la llegada de Pedro de Olavide, las ideas ilustradas, o no existen, o eran tan secretas que no se apre$\operatorname{cian}^{13}$. En la segunda mitad, España fue escenario de intensas controversias ideológicas influidas por las ideas revolucionarias de los ilustrados; también de profundos enfrentamientos entre los sectores conservadores de la Iglesia Católica y las manifestaciones racionalistas, anti escolásticas y anti aristotélicas que se apoyaban en el sensualismo de Condillac y de Bonet; y el materialismo francés de Julien Offray de La Mettrie, Denis Diderot, Paul Henri Thiry d'Holbach y Claude-Adrien Helvétius (Helvecio). Buen ejemplo de ellos son las palabras del matemático, teórico de la música y jesuita Antonio Eximeno: «nadie me pareció más perspicaz que Descartes, Locke y Leibniz a los cuales suelo llamar

11 Fue creada en Madrid en 1582, tuvo de primer director al luso - Portugal pertenecía entonces al reino de España - Juan Bautista LABAÑa, y en ella colaboraron Pedro Ambrosio de ONDÉRIz, quien tradujo en 1584 la Perspectiva y Especularia, de Euclides; Juan Cedillo Díez, quien tradujo los seis primeros libros de los Elementos, también de Euclides. Entre los alumnos, destaca Luis CARDUCHI, que también tradujo los Elementos en 1637 y fue de los primeros en mencionar los logaritmos; y Ginés de Rocamora y Torrano. El Colegio Imperial, fundado por los jesuitas, incorporó la Academia de Matemáticas (1624-1761).

12 El Colegio Imperial fue creado en franca oposición a las Universidades, de acuerdo con José Simón Díaz, Historia del Colegio Imperial de Madrid. Madrid, Consejo Superior de Investigaciones Científicas, Instituto de Estudios Madrileños, 1952-1959.

13 Ver Thomas Munck, Historia social de la Ilustración, Barcelona, Crítica, 2001. 
triunviros de los asuntos metafísicos, y Malebranche, Condillac y Bonnet». En su contra los aristotélicos, llega a afirmar jocosamente al tratar de los axiomas de la matemática: «sería mejor, por consiguiente, derogar este tipo de proposiciones y en su lugar escribir, al comienzo de todas las ciencias, ésta sola: de las proposiciones aristotélicas nada se deduce» ${ }^{14}$. $\mathrm{O}$ cuando propone su plan de renovación de estudios en España escribe: «Las proposiciones de los aristotélicos bien ordenadas están compuestas al revés o partidas transversalmente: a saber para nuevas escuelas deber proponerse nuevos maestros, que sepan, y que dada a ellos la libertad de enseñar que conviene, se juzguen a salvo y protegidos de las insidias de estos aristotélicos».

De hecho, la Ilustración fue la universalización de la crítica racional al conjunto de creencias heredadas de los siglos anteriores y a los fundamentos ideológicos que defendían la sociedad estamental.

En España, la importancia e influencia de las ideas ilustradas fue bastante diferente por el papel de la Iglesia Católica dentro del Estado, desde siglos antes. Durante la mayor parte de la edad Moderna, las monarquías españolas estuvieron más interesadas en la defensa de la Iglesia Católica y su utilidad para defender las estructuras imperiales y supranacionales que servían de beneficio a la metrópoli.

De suerte que mientras en Europa, durante el siglo XVII, se empezaba a elaborar una enseñanza superior útil y práctica, y se operó un desarrollo científico importante, dando origen a la gran ciencia que hizo desarrollar las sociedades industriales en el siglo XVIII, en España nos interesaban las disquisiciones escolásticas y nos especializábamos en la mixtura entre ciencia y religión ${ }^{15}$.

Por ello, los trabajos y descubrimientos realizados por Pedrayes, fueron en aquella época mucho más valorados en Francia y otros países europeos que en España. Como ya dijimos anteriormente hay que acudir a las Actas de la Academia de Ciencias de París para ver como se le califica de sabio ${ }^{16}$.

14 Antonio Eximeno en De studiis philosophicis et mathematids instituendes, Madrid, [s.n.], 1789, págs. 35-36.

15 Baste recordar que matemáticos religiosos como Pedro CiRuelo, a pesar de sus creencias, hubieron de escribir alegatos contra las supersticiones, la astrología y la superchería.

16 El propio Méchain en carta de 26 de 1803 dice: «Nuestra clase que conserva el nombre de $1^{\text {a }}$ clase del Instituto para las ciencias matemáticas y físicas, tendrá cien correspondientes y sobre este número faltan actualmente treinta y dos. Va a procederse inmediatamente a su elección que será como la de los miembros residentes y por la clase sola. Parece resuelto que todos los antiguos miembros extranjeros de la Comisión de pesos y medidas serán nombrados correspondientes por deseo general de la clase, así espero tener bien pronto la satisfacción de nombraros mi colega del Instituto» en Javier RUBio VIDAL, Un matemático asturiano casi olvidado, Agustín de Pedrayes. Discurso de recepción en IDEA, 20 de diciembre de 1950; contestación de Ignacio Patac Pérez, Oviedo, IDEA, 1951. 
Agustín de Pedrayes empieza estudiando Filosofía, Teología y Leyes a pesar de que sus orientaciones de estudio se dirigían hacia la matemática. Ello se debe a que en España no eran posibles estudios oficiales de la ciencia al margen de las instituciones religiosas.

La relación entre religión y poder político, tan estrecha, ha sido una de las principales causas del atraso de la ciencia española, al menos en las instituciones universitarias. Desde la Edad Media, de acuerdo con las ideas de Eusebio de Cesárea, difundidas por San Jerónimo, se consideraba que todo lo que sucedía en el mundo terrenal respondía a un plan divino, dentro del cual los hombres debían cumplir sus designios y los historiadores descifrarlos. En el plano ideológico de estos principios, lo que realmente introduce la Ilustración y la Revolución Francesa cambia el esquema y plantea la posibilidad de cambiar la Historia por los propios hombres.

La Reforma Católica o Contrarreforma contra la Reforma protestante de Martín Lutero, tuvo en el Estado español su más valioso defensor, desde el pontificado del Papa Pío IV en 1560, hasta el fin de la Guerra de los Treinta Años, en 1648. Sus armas más poderosas fueron la Inquisición y la censura ${ }^{17}$ de libros y su expansión llevó al clero español a evangelizar los pueblos indígenas, mediante un sinfín de misiones, cuya finalidad más importante, desde Trento, fue la defensa de unos principios ideológicos en nada útiles para el desarrollo de la nación.

De otro lado, las guerras de religión ${ }^{18}$ en las que se embarcó el Estado español, supusieron un incremento de la deuda del Estado que traería como consecuencia el atraso de buena parte del siglo XVII y la primera mitad del XVIII.

La Contrarreforma trajo consecuencias tan nefastas o peores que la misma Reforma, en particular contra la ciencia y los hombres de ciencia. La persecución de la Inquisición sobre científicos y publicaciones fue extrema. Personajes tan relevantes como Olavide, Gabriel Ciscar, Jovellanos y el propio Pedrayes hubieron de sufrir persecución, destierro, prisión y exilio. No es de extrañar por tanto, que Pedrayes figurara entre los firmantes de la solicitud de

17 Las obras de pensadores extranjeros eran retenidas: se les hacía un proceso y se las juzgaba. Los libros impresos en España tenían seis censuras antes de su edición, siendo supervisadas por franciscanos, o dominicos. En el siglo XVIII las obras prohibidas eran por lo general de filosofía o teología, mientras que los tratados científicos empiezan a circular con mayor libertad. Aunque con algunas excepciones, aumentaron el número de traducciones, especialmente del latín y del francés.

18 Si bien, propiamente se conocen así las que se dieron en Francia (1562-1598) entre católicos y hugonotes (calvinistas), nos interesa aquí los enfrentamientos entre la Reforma y la Contrarreforma y los Estados que las representaban. 
disolución del Tribunal de la Inquisición presentada el 13 de febrero de 1813 a las Cortes de Cádiz.

Con la sola eliminación del Tribunal de la Inquisición con el especioso nombre de Santo Oficio, la ciencia en España hubiera podido progresar con mucha más celeridad.

Evidentemente había que tener una gran potencia intelectual para proponer desarraigar ideas preconcebidas y comúnmente admitidas. Sin embargo las ideas de los científicos más sobresalientes siempre florecían por encima de la rutina de los seudocientíficos y teólogos tradicionales que pretendían conservar la tradición por encima de todo. Y también que el tiempo de retraso de esa imposición, supuso un retraso en el desarrollo de la ciencia y de las sociedades correspondientes. Y aunque en la antigüedad, el desarrollo de la astronomía y la matemática se hizo en torno a los templos, los derechos laicos de la ciencia no dejaron de ser exigidos hasta nuestros días: desde la Academia y el Liceo griegos, hasta las academias militares de nuestro siglo XVIII, o las academias científicas de los siglos XVII, XVIII y XIX. La relación entre los poderes religiosos y políticos y las instituciones científicas, fue, por lo general, de lucha encarnizada.

Las persecuciones de los hombres de ciencia se recrudecieron con la aparición de la Inquisición, con el fin de eliminar las herejías en los territorios dominados por la Iglesia Católica. Al igual que la de Alejandría, las bibliotecas se quemaban, como la de Granada, ordenada por Cisneros, en la que se perdieron importantes obras árabes de medicina. O la de Pedrayes, ejecutada por las tropas francesas en el Observatorio astronómico de Madrid o en la Academia de Artillería de Segovia ${ }^{19}$.

Igual que soportaron los rigores y la censura del Santo Oficio, Feijoo, Sarmiento y muchos otros, hubo de hacerlo Agustín de Pedrayes. Conocía los más importantes descubrimientos de la matemática de entonces por reproducciones que, en ocasiones, superaban la censura religiosa.

\section{El origen de la ciencia moderna en España}

El interés por la ciencia y en particular por la matemática, de los hombres ilustrados del siglo XVIII, tiene su base en las necesidades de la economía del Estado Español para la náutica, la astronomía, la mineralogía y la medicina, tal como dijimos. La ciencia española del Renacimiento alcanza un mayor de-

19 La Academia de Artillería de Segovia, fue fundada el 7 de enero de 1763, fruto de la fusión de las Academias de matemáticas de Barcelona y Cádiz, desaparecidas en 1760. 
sarrollo con el descubrimiento de América, y el interés por colonizar culturalmente el Viejo Continente. En 1532 aparecen los primeros libros impresos en el Nuevo Mundo ${ }^{20}$ y se funda la primera de sus universidades: la de Santo Domingo (1538).

Esa ciencia española, desde el Renacimiento tardío o Cinquecento, está muy ausente de importantes matemáticos que puedan compararse con los algebristas italianos. Sin embargo, la astronomía, la náutica, la metalurgia y las ciencias naturales alcanzan su mejor momento.

La producción astronómica en los orígenes del Renacimiento está centrada en las tablas y almanaques que tenían usos muy variados: astrológicos, náuticos y propiamente astronómicos. Fue importante el judío salmantino Abraham Zacuto (1452-c. 1515)21, quien había escrito en hebreo el Hibbur ha-gadol (El gran tratado), unas tablas astronómicas posteriormente transcritas a varias lenguas $^{22}$. Zacut fue posteriormente expulsado de España. Se le atribuye la invención de un astrolabio de cobre para determinar la altura del Sol, construido así con el fin de simplificar su uso para la náutica. También de gran interés para la cosmografía, es Antonio de Nebrija (1441-1522)23, más conocido como filólogo que como científico. Había estudiado matemática en Salamanca con Nicolás Polonio o Apolonio (c. 1456), primer catedrático de astrología de dicha Universidad. Junto a sus obras astronómicas, Cosmografía, la Tabla de la diversidad de los días ${ }^{24}$ y otros tratados sobre metrología, se conoce a Nebrija por haber medido el grado de meridiano terrestre entre Mérida y Salamanca, obteniendo el valor de 62,5 millas, equivalentes a 62.500 pasos geométricos con arreglo al pie antiguo español.

El mayor interés práctico de la ciencia entonces fue la astronomía, por su utilidad para la náutica. Los Reyes Católicos crean la Casa de Contratación $(1503)^{25}$ y buscan con las personas más instruidas y prácticas en las técnicas

20 A. A. M. Stols, Antonio de Espinosa, el segundo impresor mexicano, México, Instituto Bibliográfico Mexicano 1962.

21 F. Cantera, El judío salmantino Abraham Zacut, Madrid, [Academia de Ciencias], 1931. Esta monografía publicada por la Real Academia de Ciencias de Madrid es muy importante. El mismo autor hizo un excelente resumen en su Abraham Zacut, Madrid, Aguilar, 1935.

22 Colón utilizó el Almanaque perpetuo de Zacut.

23 A. Cotarelo, Nebrija científico; disertación pronunciada en la Universidad Hispalense, Madrid, Imp. de Editorial Magisterio Español, 1947; L. Vigil y P. Ruz AızPIRI, Nebrija en el campo de la ciencia, Madrid [s.n.] 1944, págs. 71-86.

24 Antonio de Nebrija, Tabla de la diversidad de los días y horas en las ciudades, villas y lugares de España y otros de Europa: que les responden por sus paralelos, Alcalá de Henares, Arnao Guillén de Brocar, 1517. <http://bdh.bne.es/bnesearch/detalle/1604195>

25 J. Pulido, El piloto mayor de la Casa de la Contratación de Sevilla. Sevilla, Escuela de Estudios Hispanoamericanos, 1950 . 
de la navegación de altura, la enseñanza de cosmografía, astronomía y náutica. Nombrarán a Américo Vespucio (1508-1512) Piloto Mayor, al cual sucedieron Juan Díaz de Solis (1512-1516), Sebastián Caboto (1518-1548), Alonso de Chaves (1552-1586) ${ }^{26}$, entre otros. La astronomía era ciencia complementaria y necesaria al internarse en el Océano.

Los astrónomos de la época buscaban facilitar la navegación en el Océano, y para ello era imprescindible enseñar a los marinos los procedimientos utilizados con el fin de encontrar el rumbo. Como era prácticamente imposible fijar la determinación de longitudes y de la ruta seguida por los barcos, los náuticos de la época idearon el sistema cartográfico de proyección que permitía un trazado sencillo de la loxodrómica y con ello poder determinar la posición de los navíos en alta mar. Para tales fines divulgaron sus conocimientos por medio de tratados prácticos, las cosmografías. Se preocuparon de las cartas de variaciones magnéticas y de las cartas esféricas o reducidas.

Para determinar las coordenadas en el mar emplearon distintos procedimientos: el de las singladuras, utilizado solamente en el Mediterráneo y mares reducidos; el de los ángulos de posición, basado en el conocimiento de la inclinación respecto del meridiano y del paralelo, con el error de tomar por tercer lado del triángulo la cuerda de un arco de la superficie terrestre y no el arco; el de los eclipses de Sol y de Luna, de difícil uso por los pocos fenómenos; el de la nordestación y noroestación de la aguja, ideado por Felipe Guillén; el de la declinación del Sol, de difícil uso por la poca exactitud de las tablas existentes; el del transporte de la hora, propuesto por Fernando Colón, poco práctico por la inexactitud de los relojes de entonces; el de las distancias de distintos astros a la Luna, que ideó Pedro Apiano ${ }^{27}$; y, finalmente, el de Pedro Ruiz de Villegas, basado en el movimiento propio de la Luna. Los intentos por resolver el problema de las longitudes se multiplicaron, pero mientras el problema de las cartas quedó resuelto con la invención de la proyección de Mercator, la determinación de las longitudes sólo fue posible cuando se aplicaba el método de los eclipses, empleado solo en tierra firme.

Como el problema seguía sin tener solución, Felipe II convocó en 1598 un concurso internacional, con premio de 6.000 ducados de renta perpetua, y otros 2.000 de renta vitalicia ${ }^{28}$. Concurrieron al mismo diversos astrónomos y

26 J. Pulido, El piloto..., págs. 607-638.

27 Se trata de Pedro BenNewitZ o BiENEwitZ, astrónomo alemán protegido por Carlos V, varias de cuyas obras fueron rápidamente traducidas al castellano. Así el Libro de la cosmografía, Amberes, [s.n.], 1548 y el Astronómico Caesareum, [Ingoldstat], [s.n.], 1540.

28 Valuín, págs. 58-61 y 219. Acisclo Fernández Vallín, consagró su discurso de ingreso en la Real de Ciencias a la Cultura científica de España en el siglo XVI (1893). 
marinos, entre ellos Galileo ${ }^{29}$, quien en 1612 propuso el método de los satélites de Júpiter; el belga Van Langren ${ }^{30}$, quien permaneció en España entre 1631 y 1634, propuso la utilización de las variaciones de iluminación de las montañas de la Luna ${ }^{31}$. A imagen de España, los Países Bajos, Inglaterra (1714) y Francia (1720), propusieron otros tantos premios a quien solucionara el problema. En 1764, obtuvo el premio del Parlamento inglés John Harrison, ya que su cronómetro, dado a conocer en España por Jorge Juan, permitía trasladar la hora al barco en cualquier estado de la mar.

Los problemas de triangulación de la Tierra tuvieron mucha relación con los estudios geométricos de Pedrayes, su estudio de las cuadraturas y su participación en las mediciones. Una buena parte de los trabajos de cálculo diferencial e integral, que Pedrayes da a la luz, tiene relación directa con problemas geométricos, como es fácil de comprobar en la edición crítica del Nuevo y universal methodo de quadraturas determinadas ${ }^{32}$. Concibe el cálculo y el álgebra como ramas de apoyatura para los estudios de problemas geométricos. Los problemas de los cálculos astronómicos para la navegación y todo lo referente a la medición eran cuestiones de su preocupación. De ahí que hubiera sido nombrado para acompañar a Ciscar en la Comisión de Pesas y Medidas de París.

Un aspecto importante a estudiar es la distancia entre las investigaciones científicas y los estudios universitarios. A partir de mediados de siglo se disminuyó la influencia de la astrología judiciaria. Los almanaques, como los de Serrano, siguieron publicándose, pero tuvieron una audiencia mucho menor. Esta inquietud en el cambio de rumbo filosófico de la ciencia, junto con los avances en la ciencia matemática, hicieron perder mucha influencia a la astrología, incluso en las Universidades y los sectores de la Iglesia Católica. Las soluciones a los problemas de la astronomía, como el tema de los paralajes, ya no se resolverán con predicciones astrológicas. Se usará la trigonometría lineal, y sobre todo la trigonometría esférica; así como el uso matemático de la triangulación por Snellius, iniciado en 1615, y el español Pedro Esquivel, cosmógrafo

29 A. MeLón, «Galileo y el problema de determinar la longitud geográfica», Estudios Geográficos, 25, 96 (1964), págs. 299-318; D. W. W ATERs, «Galileo and longitude: fundamental contributions to a fundamental problem», Physis, 6, 3 (1964), págs. 287-302; correspondencia de Galileo especialmente los años 1616-1617; José María LóPEz PiñEro, «Galileo en la España del siglo XviI», Revista de Occidente, 40 (julio 1966), págs. 99-108.

30 A. DE Smet, Dictionary of Scientific Biography, editado por Charles Coulston Gillispie. Nueva York, Hijos de Charles Scribner, vol. 8, 1973, págs. 25-26.

31 Van Langren durante su estancia en Madrid preparó el mapa de la Luna. A. Hidalgo, «La selenografía y los jesuitas», Urania, 9 (1969), págs. 269-270.

32 Agustín Pedrayes y Foyo, Nuevo y Universal Método de cuadraturas determinadas (Ms. 8668, Biblioteca Nacional de España, 1777). 
de Felipe $\mathrm{II}^{33}$, quienes idearon el método de la triangulación, lo que permitió a Picard ${ }^{34}$ medir el arco comprendido entre París y Amiens en 1670, con un valor del radio terrestre casi sin error.

Merced a la colaboración con los científicos franceses en la medición del cuadrante de meridiano, cuestión que resolvió el problema de la figura de la Tierra, nuestros matemáticos se habían incorporado plenamente a la astronomía moderna y al heliocentrismo. En España son cada vez más amplios los sectores que defienden el heliocentrismo, unos como hipótesis y muchos como teoría cierta, y consideran manifestación física el sistema copernicano. En 1773, Gregorio de Mayans y Síscar escribe al polaco Samuel Lutero Geret de Torun ${ }^{35}$ una carta $^{36}$, en donde expone el desarrollo del copernicanismo en España, si bien desde Jorge Juan, Feijoo, Bails, Mutis en América, Tomás Mauricio López, José de Mendoza y Ríos, etc., son muchos los defensores, o críticos como el jesuita Juan Andrés, quien señala la contumacia en el error al afirmar:

En verdad, el sujetarse a sistemas y el sostenerlos obstinadamente ha cerrado el paso por largos siglos al descubrimiento de muchísimas verdades y a los progresos de las ciencias. Gracias al gusto literario de este siglo... ya se han roto aquellos grillos que nos hacían esclavos del error sin dejarnos ir en busca de la verdad ${ }^{37}$.

\section{Como dice Juan Vernet Ginés en su Historia de la ciencia española:}

Parece, sin embargo, indudable que en la primera mitad del siglo de las luces, los españoles tuvieron conciencia muy clara de la incapacidad de las universidades para ponerse al corriente del desarrollo de la ciencia contemporánea. La única diferencia respecto al exterior radicaba en que, mientras en el resto de Europa ya

33 R. DE Manjarrés, Intervención de España en la determinación de la figura de la Tierra, Sevilla, [s.n.], 1917, págs. 163-170.

34 M. PiCard en sus Principios (1687) formula la ley que rige los movimientos de los astros. Según las predicciones de Newton, contrarias a las de Descartes, la Tierra no tenía una figura esférica, sino la de un elipsoide de rotación achatada por los polos, idea corroborada por las observaciones que Richer había hecho con péndulo a distintas latitudes, al observar su periodo. En contra estaban los Cassini I y II, La Hire y Maraldi, quienes, de acuerdo con medición del arco de meridiano entre Dunquerque y Colliure, afirmaban en 1718, que el achatamiento se producía en el ecuador. Para dilucidar la cuestión, se planteó realizar la medida de dos arcos de meridiano lo más cercanos posibles al polo y al ecuador. Y el gobierno francés decidió enviar dos expediciones: una a Laponia en el norte de Finlandia, dirigida por Maupertuis, y otra al Perú, dirigida por Louis Godin.

35 Profesor de Filosofía y Teología y autor de un calendario para 1760.

36 Editada por Vicente Peset Llorca, Acerca de la difusión del sistema copernicano en España, Salamanca, [s.n.], 1965, págs. 309-324.

37 Clelia Pighetтi, «Un gesuita difensore del Galilei», Archives Internationales d'Histoire des Sciences, 15 (1962), págs. 285-290. 
desde los inicios de la ciencia experimental habían descubierto esta realidad y habían paliado el defecto mediante la creación de instituciones independientes, España continuó ceñida a sus universidades y aun creó nuevas a todo lo largo y ancho de sus dominios, por ejemplo, la de Manila (1611), La Habana (1728) y Caracas (1721), en muchas de las cuales faltaban estudios adecuados para las matemáticas o la física, consideradas con frecuencia como disciplinas introductorias al estudio de la medicina, y, por consiguiente, hubo que admitir normas de convalidación para las mismas (1787). Porque estas instituciones, incluso las de nueva planta, como la de Cervera, creada (11-5-1717) tras la supresión de la de Barcelona, parecían tener como único fin la formación de jurisconsultos, teólogos y, en menor grado, médicos (diecisiete cátedras de los dos derechos; trece de filosofía y teología, etc.; seis de medicina, y una de matemáticas en Cervera. Y esta última materia careció de importancia hasta que se hizo cargo de ella, en 1758, Cerdá) ${ }^{38}$.

Entre las causas del atraso de la ciencia y la matemática española en el siglo XVIII, no solo está la Universidad. También influyeron las creencias de que «todo estaba ya descubierto», o que la tradición había que respetarla de forma sagrada. El propio Jovellanos decía que: «los desaguisados de la ciencia española se debían al aristotelismo y la escolástica imperante».

Por ejemplo, la Universidad de Salamanca se manifestaba aún en $1771^{39}$ partidaria decidida de Aristóteles, negando que Newton, Gassendi o Descartes puedan entrar en las aulas católicas. No podían entrar porque «giran sus sistemas sobre principios voluntarios, de que deducen conclusiones también voluntarias e impersuadibles, como diremos luego». Algunas órdenes como los carmelitas descalzos, consideraban conveniente leer, «con las precauciones que se deben observar en el estudio de algunos de ellos», nada menos que los mismos autores que condenaba la Universidad de Salamanca: Gassendi, Descartes, Newton, Leibnitz, Condillac, etc.

Todo ello, a pesar de las palabras de Menéndez y Pelayo ${ }^{40}$ quien afirmaba que la Universidad de Salamanca y su Colegio de Filosofía fueron, a fines del siglo XVIII, un foco de ideología materialista ${ }^{41}$ y radicalismo político, del que sa-

38 Juan Vernet GinÉs, Historia de la ciencia española, Madrid, Instituto de España, Cátedra Alfonso X el Sabio, 1975.

39 M. y J. L. PESET, El reformismo de Carlos III y la universidad de Salamanca. Plan general de estudios dirigido a la Universidad de Salamanca por el Real y Supremo Consejo de Castilla en 1771, Salamanca, Universidad, 1969.

40 Marcelino Menéndez Y Pelayo, Historia de los heterodoxos españoles, capítulo V, libro VI, Madrid, La Editorial Católica, 1978, pág. 880.

41 Las ideas enciclopedistas entraron en Salamanca, bajo la influencia de la librería francesa de Alegría y Clemente, donde Juan Meléndez Valdés leía el Derecho de gentes de Vattel y el Espíritu de las Leyes de Montesquieu. 
lió la mayor parte de los legisladores de 1812 y conspiradores de 1820: Manuel José Quintana, Bartolomé José Gallardo, Diego Muñoz Torrero y Ramón de Sala y Cortés. Menéndez y Pelayo dice:

¡A tal grado de miseria había llegado la filosofía en la patria de Suárez! Y por lo mismo que parecían fáciles a la comprensión las groserías empíricas, propagáronse como la lepra, y fueron la única filosofía de nuestros literatos y hombres políticos en los primeros treinta años del siglo XIX. Esa es la que propagaron Reinoso en Sevilla, el P. Muñoz en Córdoba, y D. Juan Justo García, D. Ramón de Salas [1] y otros muchos en Salamanca, cuya Universidad, y especialmente el Colegio de Filosofía, eran, a fines de la decimoctava centuria, un foco de ideología materialista y de [pág. 290] radicalismo político. De allí salieron la mayor parte de los legisladores de 1812 y de los conspiradores de 1820.

Una cuestión polémica fue la relación de nuestros científicos con sus coetáneos franceses con anterioridad a la Guerra de la Independencia. Fueron intensas, fluidas y de gran colaboración, a pesar de tener que soportar la oposición de los ultramontanos que los acusaron de traidores a la patria y de no defender los intereses de la nación.

Buen ejemplo fueron Jorge Juan y Antonio de Ulloa con Louis Godin y La Condamine en la expedición al Perú.

La historiografía al respecto tiene un propensión excesiva a simplificar el problema como una manifestación del enfrentamiento entre afrancesados y patriotas. La Guerra de la Independencia y sus barbaridades hicieron desaparecer multitud de documentos que nos hubieran ayudado a clarificar más esta cuestión, entre ellos varios de Pedrayes. Las instituciones científicas y su documentación estuvieron desde el inicio de la Guerra bajo control del Ministerio del Interior creado por José I. Y aunque algunos colaboraron con las nuevas autoridades políticas, no es menos cierto que muchos hombres de ciencia expresaban sus relaciones con Francia a través del interés por los científicos ilustrados de aquel país, al margen de los poderes políticos de Francia y España.

Tuvieron gran importancia las Academias. La primer Academia de Ciencias española, que ha llegado hasta nuestros días, fue la Real Academia de Ciencias y Artes de Barcelona (1764), cuyo origen estuvo en la «Conferencia Físico Matemática Experimental» ${ }^{42}$.

42 J. Iglesies Fort, «La Real Academia de Ciencias Naturales y Artes en el siglo XVIII», Memorias de la Real Academia de Ciencias y Artes de Barcelona; época 3, XXXVI (1964), no 707, págs. 1-635. 
Por impulso del Gobierno y más tarde de la iniciativa privada se crearon las academias matemáticas de Barcelona (1736-1760) y de Cádiz, que fueron suprimidas cuando se funda la Academia de Artillería de Segovia (7-1-1763) a la cual pasaron sus libros y pertenencias; el Real Seminario de Nobles de Madrid (21-9-1725), que se unió en 1786 a la Real Casa de Caballeros Pajes ${ }^{44}$ cuya creación había tenido lugar mucho antes; los Reales Estudios de San Isidro creados el 27-2-1767 y destinados a compensar el colapso del Colegio Imperial en el momento de la expulsión de los jesuitas.

Por su importancia, se deben considerar las Sociedades Económicas de Amigos del País, que aparecen durante el reinado de Carlos III. Iniciadas con la Vascongada (1765), siguen las de Madrid, Barcelona, Zaragoza, Valencia, Sevilla, Palma, Tudela, Segovia, Oviedo, etc. Muchas de ellas, según Sempere y Guarinos, «apenas han dado más muestras de su existencia que la de haberse anunciado su fundación en la Gaceta y conservarse su nombre y los de sus directores y secretarios en la Guía de forasteros...» ${ }^{45}$. Algunas tuvieron vida activa, en primer lugar la Vascongada, gracias a los nobles que en cierto modo intentaban demostrar el que su clase era útil al país ${ }^{46}$, y debido a que el gobierno abría las puertas de la nobleza a los hombres de empresa (18-3-1783) e introducía reformas favorables a los artesanos y menestrales en las prerrogativas de hidalguía (3-11-1770).

\section{La historiografía sobre la ciencia en el siglo XVIII en España}

La postergada Historia de la Ciencia tuvo en España después de las guerras y avatares del siglo XIX, un cierto brote a fines del mismo siglo, merced a los premios instituidos por entidades públicas, como la Biblioteca Nacional o la Real de Ciencias; etapa en la que se estimula el estudio de la ciencia. Además de Menéndez y Pelayo y de Fernández de Navarrete, Manuel Rico y Sinobas, catedrático de física superior de la Universidad de Madrid, colabora en la edición de Los libros del saber de astronomía, de Alfonso X el Sabio, y elabora un catálogo de las auroras boreales observadas en España durante los siglos

43 P. A. Pérez Ruiz, Biografía del Colegio-Academia de Artillería de Segovia, Segovia: Academia de Artillería, 1960.

44 Constituida en época de Felipe el Hermoso.

45 Juan Sempere y Guarinos, Ensayo de una biblioteca española de los mejores escritores del reinado de Carlos III. Reproducción facs., Valladolid, Consejería de Educación y Cultura, 1997.

46 A. DE Otaola, «Nobleza comerciante y Sociedades de Amigos del País», Boletín de la Real Sociedad Vascongada de Amigos del País, 21 (1965), págs. 131-150. 
XVIII y XIX. Felipe Picatoste y Rodríguez, progresista que alcanzó su máximo predicamento político durante la «gloriosa», enseñó como sustituto de matemáticas en el Instituto de San Isidro (1852-1857), y fue periodista y polígrafo; escribió diversos manuales de distintas disciplinas, y ganó un premio de la Real de Ciencias (1881) con la obra Calderón ante la ciencia ${ }^{47}$ y otro de la Biblioteca Nacional con sus Apuntes para una biblioteca científica del siglo $X V I^{48}$ (Madrid, 1891). Picatoste escribió en 1862 un Vocabulario matemático etimológico ${ }^{49}$. Con mayor rigor crítico están las obras de Acisclo Fernández Vallín y Bustillo, catedrático de matemáticas. Vallín, consagró su discurso de ingreso en la Real Academia de Ciencias a la Cultura científica de España en el siglo XVI ${ }^{50}$ (1893). Como otros trabajos de Historia de la Ciencia reposa en el limbo de los justos, depósito de numerosos catálogos y bibliografías, y es una suma que refleja las numerosas lecturas que realizó. Y a caballo entre los siglos XIX y XX están los discurso de Julio Rey Pastor (Discurso de apertura curso académico 19131914, en Oviedo sobre los matemáticos españoles del siglo XVI), Echegaray en 1866, José Antonio Elizalde (Los progresos de las matemáticas desde los tiempos más remotos), Enrique Fernández y Echevarría (Discurso de apertura curso académico 1908-1909, sobre la medida, en Oviedo), José María Frontera y Aurrecoechea (Discurso de apertura curso académico 1915-1916, en Oviedo sobre el papel de la Universidad), José Mur y Ainsa (Discurso de apertura curso académico 1900-1901, en Oviedo sobre historia de la matemática y también en el curso 1910-1911), Tomás Rivero (Discurso de apertura curso académico, en Oviedo del 1 de octubre de 1852), José M. Vijande y Fernández Luanco (Discurso de apertura curso académico 1917-1918, en Oviedo sobre el papel de la Facultad de Ciencias), y otros.

Por tanto, una segunda reflexión: lo cierto es que los estudiosos de las facultades de Humanidades desprecian la historia de la ciencia por considerarla específica de los investigadores del mundo científico, soslayando quizá con ello su desconocimiento de los dominios de las ciencias. Y que los correspondientes de las facultades de Ciencias desprecian ampliamente el pasado, enfrascados en sus resultados inmediatos.

\footnotetext{
47 Felipe Picatoste y Rodríguez, Calderón ante la ciencia, Madrid, Imprenta de la viuda e hijos de Aguado, 1881.

48 Felipe Picatoste y Rodríguez, Apuntes para una biblioteca científica española del siglo XVI, Madrid, Ollero y Ramos, 1999.

49 Felipe Picatoste y Rodríguez, Vocabulario matemático etimológico, Madrid, Imprenta Aguado, 1872.

50 Acisclo Fernández Valuín, Cultura científica de España en el siglo XVI. Discurso leído en la Real Academia de Ciencias Exactas, Físicas y Naturales. Contestación de Miguel Merino (1894).
} 
Los matemáticos españoles de esa época, en particular de la segunda mitad del siglo, hicieron gala, en sus escritos y en las instituciones en las que trabajaron, de amplia cultura matemática.

Todo lo cual contrasta con la imagen que se nos ha ofrecido desde la historiografía del siglo XIX y las tendencias románticas ${ }^{51}$. El discurso de ingreso en la Academia de Ciencias de José de Echegaray ${ }^{52}$ es un buen ejemplo de ello y de estos juicios y argumentos exhibidos. El discurso tuvo réplicas inmediatas, entre otras cosas por la afirmación de que

[...] si prescindiendo de aquellos siglos en que la civilización arábiga hizo de España el primer país del mundo en cuanto a la ciencia se refiere, sólo nos fijamos en la época moderna, y comenzamos a contar desde el siglo xv, bien comprendéis que no es ésta, ni puede ser ésta en verdad, la historia de la ciencia en España, porque mal puede tener historia científica, pueblo que no ha tenido ciencia», porque en España «no hubo más que látigo, hierro, sangre, rezos, braseros y humo...; la ciencia matemática nada nos debe: no es nuestra; no hay en ella nombre alguno que labios castellanos puedan pronunciar sin esfuerzo.

La primera réplica se debe a Picatoste ${ }^{53}$, que recogía extensos pasajes de la Respuesta a la pregunta ¿qué se debe a España?, del abate Denina ${ }^{54}$, leída en la Academia de Berlín en 1782 y añadía algunas consideraciones sobre la oportunidad de lo expresado por Echegaray, afirmando que sería conveniente algún elogio de lo poco existente, o bien establecer «la causa y el remedio a este hecho, buscar su compensación y proponer medios para que no se esterilicen los esfuerzos de los españoles». Desde su punto de vista, Picatoste cree que «la gran desgracia de este país consiste en que sus hijos, lejos de defenderle, le acriminan; lejos de glorificarle, le culpan y ayudan a renegar de un pasado en que hay seguramente mucho bueno, que nos es desconocido, porque no queremos conocerlo».

51 El romanticismo enalteció el sentimiento nacional y el rechazo a las influencias extranjeras de la matemática sublime. Véase S. GARMA, «Los matemáticos españoles y la historia de las matemáticas del siglo XVIII al XIX». Actas del Primer Congreso de la Sociedad Española de Historia de la Ciencia. Madrid, [s.n.], 1978

52 José Echegaray Y EizaguirRe, matemático y dramaturgo, fue partidario de la Gloriosa (La Revolución de 1868 o La Gloriosa, también conocida por La Septembrina) y durante ese período recorrió con éxito el escalafón de la política, llegando a ser Ministro de Fomento. Radical, partidario de la libertad de enseñanza, protector más tarde de la Institución Libre de Enseñanza, sacó a relucir su propia ideología en su discurso de entrada en la Real de Ciencias (1866) titulado Historia de las matemáticas puras en nuestra España, Murcia, Colegio de Ingenieros de Caminos, Canales y Puertos, 2007.

53 Publicada en Las Novedades, Madrid, 17 de marzo de 1866.

54 Carlo Giovanni Maria Denina (1731-1813) fue un historiador italiano. La referencia del libro: Carlo Giovanni Maria Denina, Respuesta a la pregunta ¿qué se debe a España?, traducido por Manuel Urqullu, Valencia, Salvador Faulí, 1786. 
La segunda réplica fue obra del periodista Antonio Sánchez Pérez ${ }^{55}$, más tarde catedrático del Instituto de San Isidro. Se limitó a recordar algunos matemáticos, que España había tenido como Jorge Juan. El discurso de Echegaray y las réplicas citadas son el antecedente de la gran polémica de la ciencia española, en que se enfrentaron las ideologías conservadoras y liberal: Menéndez y Pelayo, Laverde, Pidal y Mon, discípulo del cardenal Zeferino González por la primera, Revilla, Azcárate y Perojo por la segunda.

Para Echegaray, partidario del movimiento regeneracionista del XIX, hasta esas fechas no hubo nada digno de mención, o muy poco, y el verdadero despegue de la ciencia en España, empezaba entonces. Así, nos dice:

Nuestro país, que aspira afanoso a su regeneración en todas las esferas, no podía ser extraño a este gran movimiento científico de Europa, y yo debo consignar que, aparte de los relevantes servicios prestados a la ciencia por esta ilustre Academia, y que por sabidos es inútil repetir, a los cuerpos facultativos, así militares como civiles, y a sus Escuelas especiales se debe ese gran adelanto en los estudios matemáticos que se nota en España de algunos años acá. No puede, en verdad, gloriarse nuestro país de ningún importante descubrimiento, porque cuando tan rezagada queda una nación, harto hace con alcanzar a las que en tres siglos la aventajan.

Y más adelante:

Mal puede tener historia científica, pueblo que no ha tenido ciencia [...]. No es la historia de la ciencia, aquí donde no hubo más que látigo, hierro, sangre, rezos, braseros y humo. Y no he tenido que referir la historia de las matemáticas, allá, para probar que no la hay aquí, y para probarlo, señores, con la elocuente voz de los hechos, demostración ruda pero firmísima, contra la cual se estrellan impotentes, sofismas, alharacas y declaraciones, he necesitado buscar la filiación de cada verdad, el origen de cada teoría, el nacimiento de cada idea, el autor de cada descubrimiento, y después los hombres que desarrollan y perfeccionan aquellos descubrimientos y teorías, formando de esta suerte la ciencia moderna en toda su magnífica riqueza; y he necesitado todo esto para poder decir sin remordimiento y sin temor: la ciencia matemática nada nos debe: no es nuestra; no hay en ella nombre alguno que labios castellanos puedan pronunciar sin esfuerzo ${ }^{56}$.

55 F. VERA, Los historiadores de la matemática española, Madrid, V. Suárez, 1935, págs. 72-76.

56 Página 16 del Discurso de José de Echegaray, Historia de las matemáticas puras en nuestra España, págs. 15-16. 
Y después:

Todos estos pueblos, entre guerras y sangre, y terribles sacudimientos, conservan entera y vigorosa su razón, y de entre el caos y las ruinas se alzan genios potentes, nobles inteligencias, profundos filósofos y grandes geómetras; y en nuestra España, invencible y poderosa, dueña del mundo nuevo, y aspirando a dominar el antiguo, tranquila, relativamente al resto de Europa, en el interior, temida fuera, con su unidad política y su unidad religiosa, sólo se conservan puros, y no siempre, la imaginación y el sentimiento; pero la razón, la facultad más noble del ser que piensa, languidece y decae, y con ella todo languidece y muere al fin [...]. Nuestra deplorable decadencia desde el Renacimiento, que fue para España más bien morir que renacer, hasta principios de este siglo [...]. Hay, señores, quien imagina, y personas ilustradas y respetables son por singular inconsecuencia de esta opinión, que la gran importancia, la verdadera utilidad, el incuestionable valor de las Matemáticas puras, sólo reside en la aplicación que de los principios abstractos de la ciencia pueda hacerse a la física, a la geodesia, a la mecánica y, principalmente, a la industria; y que toda verdad científica, por elevada que sea, a la que no corresponda una utilidad práctica, y por decirlo así tangible, es vana gimnasia de la razón, fugaz relampaguear de la fantasía, juego pueril que para nada sirve, descubrimiento estéril que, sin daño del bien común, pudo quedar algunos siglos más envuelto entre las sombras, de las que le arrancó por caprichoso entretenimiento algún desocupado geómetra ${ }^{57}$.

En resumen, Echegaray no conoce la importancia de Pedrayes porque no estudió suficientemente la documentación existente en España (Universidad Complutense de Madrid, Biblioteca Nacional de España); no leyó los manuscritos de Pedrayes; no conoció las Actas del Congreso de pesas y medidas, esencial hoy; ignoró la correspondencia de los matemáticos del siglo XVIII...

Desde que se inicia la polémica sobre la ciencia española, entre los negadores de sus avances estuvieron los liberales decimonónicos. Y otro dato es que no se habían leído los textos matemáticos originales a que se referían, o si los habían leído no los habían entendido. Es decir, o no los conocían o no los asimilaron.

Ahora bien, si buscamos estudios y enseñanzas matemáticas durante el siglo XVIII en las Universidades españolas, la conclusión es clara: hubo muy poco. La Universidad era un colchón acrítico que obedecía los designios divinos, estaba dedicada a la teología y al derecho canónico, en una época en la que

57 Página 17 del Discurso de José de Echegaray, Historia de las matemáticas puras en nuestra España. 
había que obedecer y sobre todo pagar a la Iglesia para evitar ser condenados al infierno.

Los estudios de matemáticas no tenían nada que ver con estas instituciones. Las enseñanzas, el estudio y la creación matemática se centraban en las Academias militares y en algunos colegios religiosos como el de los jesuitas y benedictinos, en parte propiciados por las exigencias de los militares y los intereses de la nobleza menor. Estos grupos sociales fueron los que abrazaron y asimilaron lecturas de los ilustrados, en particular de los franceses, y promovieron tales inquietudes. Entre ellos, tuvieron gran influencia los filósofos sensistas ${ }^{58}$ y materialistas franceses.

Los estudios de historia de la ciencia en España gozaron de numerosos tópicos que contaron con la aquiescencia de algunos científicos españoles, según los cuales no hubo actividad científica importante ni descubridores de grandes teorías en la cultura de la sociedad española anterior al siglo XIX. Así se mantuvo en el mayor de los olvidos a la Historia de la Ciencia española dentro de la Historia de España con argumentos más bien ideológicos.

Así por ejemplo, basta recordar una de sus primeras manifestaciones con Juan Farreras, Bibliotecario Mayor del Rey Felipe V, quien en 1723 ordena a los funcionarios no hacer reseñas de las obras publicadas en España para remitirlas a los periódicos franceses. Y se opone diciendo que esa divulgación era inútil dado que no contenían ninguna «cosa singular, ni invención, ni descubrimiento nuevo, que era lo que los PP. de Trevoux habían ofrecido publicar» ${ }^{59}$.

\section{Ciencia y publicaciones. La propagación de la ciencia}

Las revistas científicas y las actas de sesiones científicas, distribuidas a través del comercio y las comunicaciones, fueron a partir del siglo XVII el sistema más importante de propagación de la ciencia. Pero la difusión y propagación de la ciencia no siempre tuvo un camino sencillo y llano. La propagación de las ideas se dificultaba por motivos políticos, religiosos, económicos, estratégicos, militares, y otros.

La contradicción entre ciencia y religión, o entre ciencia y los más importantes poderes de la época, fue una constante a lo largo de la Edad Moderna. Así fue, por ejemplo, la prohibición a Monge (1768) de publicar los resultados

58 Sensualismo asignado al filósofo Condillac.

59 Juan Sempere y Guarinos, Ensayo de una biblioteca española de los mejores escritores del reinado de Carlos III, op. cit, pág. 19. 
que había obtenido («con el fin de no ayudar a los extranjeros a capacitarse rápidamente en la construcción»); los motivos religiosos (prohibición de ventas de libros a gentes de distinto credo), fueron algo muy extendido desde la misma Edad Media; y los motivos económicos, generalmente tuvieron una estrecha relación con el espionaje.

En el Renacimiento, el plagio fue frecuente; razón por la que los ingenieros y hombres de ciencia, guardaban celosamente sus métodos de trabajo. Y como consecuencia lógica apareció en gran escala el espionaje industrial. Si los plagios eran de materias sin aplicación práctica, o más abstractas como la matemática, el plagio no tuvo tanta oposición. Los teoremas de Guldin sobre áreas y volúmenes de los cuerpos de revolución o la ley de los senos expuesta por Descartes, fueron copiados respectivamente de las obras de Pappus y de Snellius, sin que aquellos fuesen citados. Recientemente un historiador norteamericano de la matemática, Carl Boyer, reproduce en su Historia de las matemáticas, obra muy citada, una tabla de multiplicar de los antiguos griegos, que aparece en una obra de Fermat sobre Diofanto, sin que se cite el origen. Muchos hallazgos matemáticos, y de la ciencia en general, se atribuyen a autores determinados, cuando en realidad fueron descubiertos por otros anteriormente.

El fenómeno conocido con el nombre de convergencia, representa en ocasiones, descubrimientos que se producen al mismo tiempo en dos lugares distintos y distantes: el disco de Newton ideado por este, pero ya experimentado en el siglo XIII; la máquina de calcular de Pascal y Leibniz; el descubrimiento del cálculo infinitesimal por Leibniz y Newton; el del planeta Neptuno por Leverrier y Adams, etc.

Evidentemente estos casos y otros de igual naturaleza se presentan de manera distinta, según la ideología, la nacionalidad del historiador y otros caracteres.

Las querellas de prioridad sobre tal o cual descubrimiento científico, disminuyen en sentido inverso al incremento del poder de los medios de difusión: «La proporción de disputas que llegó al 92 por ciento durante el siglo XVII, fue del 72 por ciento en el siglo XVIII, del 50 por ciento en la segunda mitad del siglo XIX y del 33 por ciento en la primera mitad del actual ${ }^{60}$.

Tuvo gran influencia para los estudios de la matemática, las Mêmoires de Trévoux (1701-1775) ${ }^{61}$, publicación de gran difusión en España a través de las instituciones de los jesuitas, que exponía las consecuencias filosófico-teológicas

${ }^{60}$ Derek John de Solla Price; J. M. López Piñero, Hacia una Ciencia de la Ciencia, Barcelona, Ariel, 1973. pág. 16.

${ }_{61}$ Índices de C. Sommervogel (3 vols., París, 1864). El título completo es Mêmoires pour l’histoire des sciences et des Beaux-Arts. 
de los más importantes descubrimientos científicos de la época. Con éxito durante el reinado de los primeros Borbones, sucumbió pocos años después de la expulsión de los jesuitas.

Aunque en el siglo XVIII la lengua vehicular de la difusión científica fue el latín, en el siglo XVII ya se escribían algunas obras en lenguas vulgares. La utilización generalizada de las lenguas vernáculas en escritos científicos tuvo lugar en el siglo XIX. De hecho en el siglo XVIII la Academia de Ciencias de Berlín publicaba sólo obras escritas en francés o en latín, pero no en alemán.

Ello se comprueba en los propios escritos de Pedrayes. Escribe en castellano y en latín el famoso Problema que presenta a las Academias europeas. Sin embargo, para la enseñanza y la difusión de las ideas científicas en la misma, utiliza el castellano, puesto que eran instituciones no universitarias, ni eclesiásticas.

Desde mediados del siglo se empiezan a traducir libros modernos de carácter científico, en especial en los centros en los que se enseñaban matemáticas.

Surgieron grupos que, motivados por la creciente actividad científica, se emplearon con interés en la polémica con colegas de más allá de los Pirineos. O bien que, como el caso de Pedrayes, presentan problemas matemáticos para su solución, a las Academias de Ciencias de otros países europeos. Todo ello hizo crecer la formación de los matemáticos de la época y aumentar el progreso evidente en los últimos decenios del siglo XVIII y los primeros años del siglo XIX, al menos hasta la llegada de Fernando VII. Se imprimieron memorias y otros trabajos de investigación que contribuyeron a mejorar el ambiente científico español. Los matemáticos profesionalizados dedicados a la enseñanza aumentaron de forma considerable, a pesar de la resistencia de teólogos y escolásticos.

Interesaba la enseñanza del Álgebra y el Cálculo infinitesimal aplicado a la Geometría; las ecuaciones diferenciales; y los desarrollos en serie de funciones. Basta ver los Programas de estudio del Seminario de Nobles referentes a la enseñanza de la matemática, elaborados por Agustín de Pedrayes, entre otros.

Con el fin de paliar el relativo atraso en matemática y otras ciencias, se intentó a mediados de siglo XVIII, la contratación de extranjeros, como fue el caso de Lagrange, quien rehusó. Vinieron algunos científicos europeos, si bien no de primera categoría, la mayoría naturalistas, químicos y técnicos. Los científicos rehusaban por el desbarajuste del Estado como analizó Enrique Moles ${ }^{62}$ al ingresar en 1934 en la Real Academia de Ciencias, para concluir:

62 Enrique Moles Ormella, Del momento científico español 1775-1825. Discurso de ingreso en 1934 en la Real Academia de Ciencias. 
Hay que buscar la causa del fracaso de los extranjeros de importación, lo mismo que el escaso éxito de las pensiones y de los centros de investigación en algo más general y de todos los tiempos... Fracasan los laboratorios oficiales de Segovia y de Madrid, como habían fracasado antes los de Vergara, subvencionados por el Rey. No fracasa el laboratorio de la Junta de Comercio de Barcelona, sostenido con recursos propios, sin intervención del Estado. El enemigo fue siempre el mismo: la Administración y la Burocracia. Los mejores planes, las intenciones mejores, los propósitos más ideales quedan destrozados contra el muro inconmovible de la rutina...

Al propio tiempo, se hizo menos rigurosa la censura de libros y otras publicaciones y aumentaron las traducciones de tratados extranjeros, en particular del francés y del latín. Y se prohibieron desde el 21 de julio de 1767, las publicaciones de pronósticos, romances de ciego, coplas de ajusticiados, y tres años antes, según Sempere y Guarinos, se habían prohibido los autos sacramentales, a los que se calificaba de «farsas espirituales» ${ }^{63}$. En el siglo XVIII la matemática en España, comienza a implantarse en Escuelas y Colegios [Real Seminario de Nobles (1725),...] y tímidamente al final en las Universidades. Los centros más avanzados eran el Seminario de Nobles, las Escuelas de Guardias Marinas de Cádiz y a fines de siglo la de Cartagena; la recién creada Academia de Artillería de Segovia (1763); los benedictinos y los colegios de los jesuitas que tuvieron una gran influencia de los filósofos sensistas y materialistas franceses. Las obras sobre náutica se suceden: atlas marítimos, tratados sobre instrumentos o procedimientos de cálculo aplicables a la navegación y a la mejora de la cartografía. Los libros de divulgación de la época tienen contenidos al unísono con los del resto de Europa.

Desde su posición de profesor de matemáticas en la Casa de Caballeros Pajes y en el Seminario de Nobles, Pedrayes intervino directamente en la extensión del conocimiento de las matemáticas conocidas en su época y de los matemáticos europeos más sobresalientes. Por un lado, a través de la enseñanza directa y de los programas didácticos; y de otro por medio de sus publicaciones.

En este sentido, Pedrayes colaboró a las publicaciones científicas de nuestro país con: Nuevo y universal método de cuadraturas determinadas. Y el Programa con nuevo método para resolver el Problema de hallar una ecuación diferencial correspondiente a otra integral. Solución al Problema propuesto, en

63 Juan Sempere y Guarinos, Ensayo de una biblioteca española de los mejores escritores del reinado de Carlos III, op. cit., vol. 1, págs. 35-36. 
versión latina y castellana. De este Programa, presentado a concurso en las Academias europeas, presenta su solución en 1805 en el Opúsculo primero.

Su contribución a la propagación de la ciencia, también se extendió a la ciencia aplicada, la ingeniería y las tecnologías. Creó dos barómetros y un comparador para disminuir el error cuando había que cotejar patrones con el metro patrón. Este último aparato lo expone en París con motivo de su asistencia a la Asamblea para determinar el Sistema Métrico Decimal en 1799, y lo desarrolla con el ingeniero francés Lenoir. Y para medir el arco de meridiano potencia la aplicación del círculo de Borda como aparato más fiable.

\section{La matemática en la época de Pedrayes}

Durante el siglo XVIII y los primeros años del siglo XIX se desarrolló la matemática española de manera considerable, por el interés de un amplio grupo de científicos españoles.

El primer tratado monográfico conservado sobre matemáticas sublimes (antes se denominaba cálculo de fluxiones o cálculo diferencial), con influencias de Lagrange, fueron las Instituciones de cálculo diferencial e integral con sus aplicaciones principales a las matemáticas puras y mixtas ${ }^{64}$, obra de José Chaix. En Segovia explicó el italiano Cipriano Vimercati, que vino a España con Carlos III. Lo explica en el Colegio de Artillería de Segovia a partir de 1764. El francés Louis Godin ${ }^{65}$ lo enseñaba a los guardiamarinas mientras fue director de la Academia de Cádiz entre 1752 y 1760. El 19-8-1796, se crea el cuerpo de ingenieros cosmógrafos, encargado del observatorio de Madrid en el que se iba a enseñar: aritmética, análisis, geometría, cálculo infinitesimal, trigonometría plana y esférica, óptica general, astronomía «sintética», astronomía práctica, construcción de cartas, meteorología, trazado de planos y observación astronómica $^{66}$, lo que hace pensar que a fines del siglo XVIII los conocimientos de matemática infinitesimal estaban extendidos y desarrollados.

El interés de todos estos profesores era el de dotar a sus alumnos de conocimientos sólidos en matemática para poder aplicarlos a materias técnicas como la náutica, la artillería, la minería,...

Durante el reinado de los dos primeros Borbones se hace común la idea de que, salvo asuntos religiosos, no deben darse por válidas las afirmaciones de

64 Solo conocemos el primer volumen dedicado al cálculo diferencial.

65 Louis Godin fue miembro de la comisión franco-española enviada a medir el arco de meridiano en el Perú, junto con los españoles Jorge Juan y Antonio de Ulloa.

${ }_{66}$ Manuel de Godoy, Memorias (Serie: Biblioteca de Autores Españoles, 88-89), Madrid, Atlas, 1965. 
los filósofos, incluidos los de filosofía natural, si no están fundadas en la razón. A pesar de que algunos ilustrados, como Jovellanos, y científicos, como Bails, tienen vigilancia del Santo Tribunal. Por ejemplo en los Diarios de Jovellanos se escribe

[Juan] Lespardat me anuncia que el cura de Somió (comisario de Inquisición) hizo a monsieur Du Gravier varias preguntas acerca de los libros de la biblioteca, en tono de dar cuidado a éste. Dígole que esté sin cuidado, pero que no descuide; vea quién entra; no permita que nadie, en tono de registrar o reconocer los libros; no entregue el inventario (como parece se solicitó ya), y que avise de cualquiera novedad $^{67}$.

Dicen que [Antonio] Tavira será inquisidor general, y aún hay quien dice que será abolida la Inquisición. ¡Oh, cuánto ganarían en ello las letras! ¡Cuánto las costumbres! Cuantos menos fuesen los hipócritas, mejor sería. El depósito de la fe estaría mejor en manos de los obispos, de donde fue arrancado, y este padrón, que sólo sufren tres pueblos católicos, sería para siempre arrancado ${ }^{68}$.

El verdadero cambio se da a mediados de siglo, por influencia de Mayans y Siscar $^{69}$, Piquer y, en especial, de Jorge Juan y Ulloa. Pensemos en Jorge Juan y Santacilia; en Antonio de Ulloa y su colaboración en la medición del cuadrante de meridiano con los franceses; en el jesuita y primer profesor de matemáticas del Real Colegio de Artillería en Segovia, Antonio Eximeno (Valencia 1729, Roma 1808), humanista, matemático y autor de un manual sobre principios científicos de la música, mucho antes de que el alemán Helmholtz escribiera su manual clásico de investigación musical ${ }^{70}$; pensemos en Ramón Ciscar, en

67 Jovellanos, Diario, viernes 4 de septiembre de 1795, Obras completas, tomo VII, Oviedo, Instituto Feijoo de Estudios del Siglo XVIII, 1999, pág. 432.

68 Diario, lunes, 10 de abril de 1797, tomo VII, págs. 708-715.

69 V. PeSET, «Gregorio Mayáns (1699-1781) y la Historia de la Medicina», Cuadernos de Historia de la Medicina Española, IV (1965), págs. 3-53.

70 La obra de Eximeno se titula Dell'origine e delle Regole della Música, colla Storia del suo progreso, decadenza e rinuovazione, Roma, Nella Stamperia di Michel'Angelo Barbiellini nel Palazzo Massimi, 1774. Por la autoría de esta obra fue conocido entre los italianos como el «Newton de la Música». Su obra matemática más conocida es Institutiones philosophicae et mathematicae aunque el pensamiento de Eximeno se suele asociar a su obra propedéutica De studiis philosophicis et mathematicis instituendis. En 1762 Eximeno comenzó a enseñar matemáticas en el Colegio de San Pablo, contiguo al Seminario de Nobles, lugar donde hubo de enfrentarse a las corrientes conservadoras que no les satisfacía que Eximeno implantara la nueva Matemática. El Colegio San Pablo era un centro de estudios superiores para estudiantes jesuitas y seglares. En 1763 preparó para la imprenta un Tratado de matemáticas, dividido en seis partes: Álgebra y Geometría Sintética, Cálculo aplicado a la Geometría, Cálculo diferencial, integral y Mecánica abstracta, Mecánica aplicada y Astronomía. El 9 de noviembre de 1763 por carta del Conde de Gazola es nombrado Profesor de 
Celestino Mutis; en Antonio Echarri, Víctor Navarro, Ramón Gago, Francisco Aragón, Juan Justo García ${ }^{71}$ y tantos otros.

Jorge Juan defiende en su obra póstuma Estado de la astronomía en Europa (Madrid, 1773) el heliocentrismo. En la expedición del Perú (1735-1744) participan La Condamine, P. Bourger ${ }^{72}$, Godin; y los españoles Jorge Juan y Antonio de Ulloa. En la obra de Jorge Juan y Ulloa, Observaciones astronómicas y físicas ${ }^{73}$, la parte matemática se debe a Jorge Juan. La obra tuvo que soportar la observación de la Inquisición, pues

[...] suponiendo el autor en su prólogo el movimiento de la Tierra conforme al sistema de Copérnico, el Inquisidor General y los calificadores... han estado a punto de suprimir el libro; pero el P. Burriel (1719-1762), jesuita, habiendo publicado un escrito para demostrar que no se habla del movimiento de la Tierra, sino como de una hipótesis, el libro ha pasado ${ }^{74}$.

Es en las Observaciones astronómicas y físicas donde Jorge Juan demuestra conocer el análisis infinitesimal: desarrolla una fórmula en que da la razón de los dos semiejes del meridiano terrestre en función de las longitudes de arcos de un minuto medidos en distintas latitudes. Su fórmula, exacta, fue obtenida con independencia de la aproximada de Maupertuis, que se deduce de la suya ${ }^{75}$. Al tratar el problema de la rectificación de la elipse de los meridianos de la Tierra, dice:

La traen varios autores que tratan de geometría sublime y de los cálculos diferencial e integral, pero las fórmulas que dan para ello sólo pueden servir cuando se buscan arcos pequeños de la curva, pues queriéndose valer de ellas para hallar todo el cuadrante de la elipse, los términos de la serie a que se reduce dicha rec-

\footnotetext{
la Nueva Academia de Caballeros Cadetes y Oficiales del Real Cuerpo de Artillería que se va a instalar en el Alcázar de Segovia, hasta abril de 1767, fecha en la que Carlos III expulsa a los jesuitas.

${ }^{71}$ Uno de los trabajos más interesantes sobre la matemática española del siglo XVIII es el de N. CuESTA Dotari, El maestro Juan Justo García, 2 vols. Salamanca, Universidad de Salamanca, 1974.

72 R. Majo Framis, La Condamine en la América Austral, Madrid, Aguilar, 1963; La Condamine, Mésure des 3 degrés du méridien dans l'hémisphére austral, Paris; Imp. Royale, 1751. Durante esta expedición fue cuando La Condamine (1740) descubrió, en Quito, que la velocidad del sonido depende de la temperatura del aire.

73 Jorge JuAn y Antonio de UlloA, Observaciones Astronómicas y Físicas hechas en los Reinos del Perú, de las cuales se deduce la figura y magnitud de la Tierra, y se aplica a la Navegación, Por D. Jorge Juan y D. Antonio de Ulloa, Madrid, Imprenta Real, 1748.

74 A. Pérez Goyena, «En el centenario de Jorge Juan, sabio marino español. Discordancias sobre D. Jorge Juan», Razón y Fe, 37 (1913), págs. 454-469.

75 Patricio Peñalver y Bachiller, Bosquejo de la Matemática española en los siglos de la decadencia: Discurso leído en la solemne apertura del Curso Académico 1930 a 1931 en la Universidad de Sevilla, Sevilla, Imprenta y Librería de Eulogio de las Heras, 1930, pág. 52.
} 
tificación disminuyen tan poco que la operación es impracticable. Con eso me ha parecido que pueden los geómetras gustar de ver el método que yo he seguido de rectificar o hallar la periferia de la elipse de la Tierra.

Miguel de Alvear ${ }^{76}$ publicó una memoria sobre Álgebra en relación con la resolución de ecuaciones. Miguel de Alvear era profesor de la Escuela de Guardias Marinas de Cádiz y militar de profesión con la graduación de coronel. En dicha Memoria recoge una investigación sobre la solución numérica de ecuaciones de grados 4, 5 y 6, mejorando la solución que hasta entonces se usaba, basada en un texto de Bezout. Alvear descartó ya en ese momento, la posibilidad de encontrar soluciones exactas para esas ecuaciones.

Por su parte, José Chaix ${ }^{77}$ que fue vicedirector del colegio de ingenieros cosmógrafos, comisario de guerra y profesor en el centro de la Inspección general de caminos, publica otra memoria, Un nuevo método para transformar series de funciones trascendentes precedido por otro método particular para las funciones logarítmicas y exponenciales. Chaix tuvo estrecha relación con los astrónomos Agustín de Betancourt y José Mariano Vallejo. Además colaboró con los matemáticos franceses Biot y Arago, junto con José Rodríguez González, para medir el arco de meridiano. Chaix, en esa memoria que publica en Madrid en 1807 desarrolla el método expuesto por Halley ${ }^{78}$ para obtener desarrollos en serie de $\log (1+\mathrm{x})$ y de $\mathrm{a}^{\mathrm{x}}$.

Chaix quiso señalar sus diferencias con los métodos de los matemáticos franceses, para lo que quiso elaborar un método que fuese puramente algebraico, esto es libre de toda consideración sobre el infinito o lo infinitamente pequeño, que le permitiese desarrollar en serie cualquier función transcendente ${ }^{79}$.

José Sánchez Cerquero publicó sobre este mismo problema varios artículos. Cerquero era ingeniero naval en 1805 y hasta 1816 fue primer maestro de la Academia de Guardias Marinas de Cartagena. Desde ese año hasta 1846 fue director del Observatorio de San Fernando. Cerquero tuvo estrechas

76 Miguel de Alvear, Memoria sobre las Ecuaciones superiores o método general de resolverlas, San Fernando, Imprenta del Real Cuerpo de Artillería de Marina, 1814.

77 José Chaix, Memoria sobre un nuevo método para transformar en series las funciones trascendentes, precedido por otro método particular para las funciones logarítmicas y exponenciales, Madrid, en la Imprenta Real, 1807.

78 Este método con menor desarrollo y distinta notación, había sido publicado en las Phylosophical Transactions de la Royal Society, en 1695.

79 Se trataba de usar una propiedad que cumpliesen las funciones para conseguir una igualdad en la que se podía sustituir la función por una expresión polinómica con coeficientes indeterminados y después por el procedimiento de igualar los términos correspondientes se calculaban los coeficientes. Este mismo método fue expuesto por Pedrayes en 1777 en su trabajo sobre las Cuadraturas. 
relaciones con los científicos ingleses, llegando a formar parte de la Royal Astronomic Society of London y de la Meteorological Society. También trabajó en el Observatorio de Greenwich. Entre sus artículos, se encuentran en la Correspondence Mathematique de Quetelet $(10,1838)$, Sur le developpement des functions exponentielles et logarithmiques y Sur une demostration nouvelle du developpement du binome. En el Periódico mensual de Ciencias de Cádiz ${ }^{30}$, se publicaron Observaciones sobre el desarrollo de las funciones en serie, empleando la diferenciación y Continuación de las investigaciones sobre el desarrollo de las funciones analíticas ${ }^{81}$.

Las aplicaciones del Cálculo infinitesimal a la Geometría fueron desarrolladas por José Mariano Vallejo en su Memoria sobre La curvatura de las líneas en sus diferentes puntos sobre el radio de curvatura y sobre sus evolutas, publicada en Madrid en 1807. Vallejo fue también profesor de matemáticas en el Real Seminario de Nobles de Madrid, fue amigo de Laplace y colaboró en la fundación del Ateneo de Madrid y en la Academia de Ciencias.

Por otro lado, José Joaquín de Ferrer, astrónomo, planteó la ecuación diferencial de Ferrer-Legendre, y publicó numerosos trabajos dedicados a la enseñanza. Como vemos, el periodo correspondiente a los años que van desde la mitad del siglo XVIII hasta el primer tercio del XIX fue fecundo para la ciencia y el desarrollo de los científicos e ingenieros españoles.

Todos estos científicos desarrollaron su trabajo en difíciles condiciones sociales y políticas. Todos estos estudiosos científicos españoles tuvieron enfrente la más encarnizada persecución, pese a lo cual pudieron brillar ampliamente, haciendo la etapa del siglo XVIII grande y próspera, científicamente hablando.

La mayor parte de los matemáticos de la época desarrollaron su trabajo de investigación y enseñanza en los colegios de jesuitas ${ }^{82}$ y benedictinos, así como en las Academias militares de Guardias Marinas. Y también en organismos particulares. El propio Agustín de Pedrayes, no se incorpora a la enseñanza e investigación en una institución universitaria, sino en el Seminario de Nobles, ya que el desarrollo científico tenía lugar en Academias y Escuelas militares, que era donde la enseñanza de la ciencia se aplicaba a las necesidades de la sociedad. Una de las características de la ciencia moderna.

$80 \quad$ Periódico mensual de ciencias matemáticas y físicas, Cádiz, [s.n.],1848.

81 José Sánchez Cerquero, «Observaciones sobre una parte de la doctrina de las secciones angulares», Periódico Mensual de Ciencias de Cádiz, no 1 (1848), págs. 35-53. Y «Continuación de las investigaciones sobre el desarrollo de las funciones analíticas», Periódico Mensual de Ciencias de Cádiz, nº 1 (1848), págs. 116-142.

82 El General de la Compañía de Jesús, P. Ricci, potenció por entonces, el fomento de la matemática en los colegios de la Compañía. 
De las influencias del desarrollo científico de la primera mitad del siglo XVIII es buena muestra la inquietud intelectual de Agustín de Pedrayes y Foyo. Tuvo una salud mediocre y por eso enfermó. Sus preocupaciones para consigo siempre tuvieron por finalidad la utilización del mayor tiempo posible dedicado a sus estudios matemáticos. Vivió para la ciencia matemática y ésta debe perpetuar su memoria. El duelo y recuerdo universal de las ciencias es hoy expresado con plena libertad; con sinceridad y con rabia por tanto olvido de tantos y tantos españoles célebres.

Podrán menospreciar la ciencia, la investigación y el saber, y seguir despreciándola con el mayor de los olvidos. Podrán centrar sus preocupaciones en darse palmadas y cantar victorias y olés donde solamente hay mediocridad. Pero mal que les pese, «la Tierra sigue moviéndose alrededor del Sol».

Propone Agustín de Pedrayes y Foyo, en 1777, un Nuevo y universal método de cuadraturas determinadas con el fin de integrar áreas limitadas por curvas, en particular por cónicas. En 1796 propuso un concurso internacional consistente en integrar una función en la que casi todos los términos son diferenciales binomias $^{83}$, que tuvo la inquietud de la comunidad matemática internacional. En el trabajo de 1777 ya aparece el esbozo del Problema que suscitará más adelante, en 1796. Dicho Problema fue señalado en nombre de S. M. Católica con tres premios de cinco mil reales cada uno, para el que en el término de un año se resolviese el problema en Francia, Alemania y España, habiéndose ofrecido el Instituto Nacional de Francia para juzgar las memorias que se le remitiesen (Moniteur número 345) ${ }^{84}$. El Secretario Perpetuo de la Real Academia de Berlín recibió una solución que fue examinada por Delambre, del Instituto de Francia, quien consideró que no satisfacía la solución correctamente.

Además de sus aportaciones teóricas a la matemática diferencial, en 1799 Agustín de Pedrayes, junto con Gabriel Ciscar y Ciscar ${ }^{85}$, fue comisionado por el gobierno español para asistir a las reuniones celebradas en París entre 1798 y 1800 con el fin instaurar el sistema métrico decimal, que tenía su fundamento en las mediciones geodésicas realizadas a lo largo del siglo XVIII.

83 Agustín de Pedrayes y Foyo, Solución del Problema Propuesto el año de 1797 / [Agustín Pedrayes]; Dado a luz por una asociación literaria, Madrid, Imprenta de la Administración del Real Arbitrio de Beneficencia, 1805 <http://bvpb.mcu.es/es/catalogo_imagenes/grupo.cmd?path=387>

$84 \quad$ El 17 de abril de 1798 se publica en la Gaceta de Madrid el concurso abierto para la resolución de dicho problema, siendo Pedrayes ganador en solitario e incitado a París.

85 J. B. RoвerT, «El teniente general de la armada don Gabriel Ciscar y Ciscar (1760-1829)», Revista General de la Marina, 158, 1960, págs. 279-289; G. CISCAR, Memoria elemental sobre las nuevas pesas y medidas decimales fundadas en la naturaleza, Madrid, Imprenta Real por Pedro Pereira, 1800. 
La matemática, hoy, admite y aplica de forma generalizada y rigurosa, como uno de sus mayores logros, las investigaciones de Pedrayes que condujeron a los descubrimientos de la integral de Riemann y al estudio de las integrales elípticas por parte de Hermite y Poincaré. Y hasta fecha muy reciente, hasta las medidas actuales con rayos láser, seguimos midiendo con el metro que ideó Pedrayes. Los títulos de su gloria no los pueden enterrar porque forman parte de la vida misma. Y la gloria de un creador como Agustín de Pedrayes, no se puede aniquilar porque la huella de su genio deberá reconocer la inmortalidad.

Como sucede con todas las contiendas militares y sus consecuencias, la Guerra de la Independencia truncó toda aquella perspectiva ilusionante.

Agustín de Pedrayes fue un magnífico enseñante que dedicó buena parte de su vida — veintidós años- a transmitir conocimientos, al tiempo que investigaba nuevas creaciones. Durante su estancia como maestro del Seminario de Nobles, elabora para ese centro de enseñanza el Programa de enseñanza de la matemática, en 1788. En él dice:

La Geometría dá las verdaderas ideas del método, de la evidencia y exactitud; y el talento geométrico no puede dexar de ser muy á propósito para adelantar en qualesquiera otras ciencias y artes á que se aplique, especialmente en aquellas que los Nobles según sus particulares destinos deben poseer con mas perfeccion. Pero al mismo tiempo que es tan importante el estudio de las ciencias Matemáticas, aun para otras profesiones; en qualquiera parte que se enseñe esta Facultad, la experiencia acredita quan corto es el número de los jóvenes asistentes á las clases que manifiestan algún talento geométrico: pues su estudio pide una atencion y meditacion en grado superior á la que se observa en la mayor parte, y son en menor número los que están en estado de sacar de él algún adelantamiento. Por la misma razon se debe juzgar que no es el mas conveniente á esta primera edad el método mas riguroso de proponer las verdades matemáticas: exigiendo este una combinacion mas complicada de proposiciones y de demostraciones, es preferible el método que demuestra las mismas verdades con mas brevedad y claridad, aunque de las proposiciones muy claras se tenga solamente el primer grado de evidencia. Los ingenios que descubran una inclinacion decidida á las Matemáticas, podrán suplir con facilidad esta falta por la lectura de varios Autores, que siempre es necesaria para hacer progresos en dichas ciencias ${ }^{86}$.

86 Agustín de Pedrayes y Foyo, Conclusiones matemáticas de arismética, álgebra y geometría, que en el real seminario de nobles de esta corte (...) baxo la dirección de su catedrático don Agustín de Pedrayes, Madrid, Imprenta de la viuda de Ibarra, 1788. 
Posteriormente colaboró con Jovellanos elaborando el Programa de enseñanza de la Matemática para el Real Instituto Asturiano de Náutica y Mineralogía.

Pedrayes, durante su etapa de enseñante y después de convaleciente, nunca dejó de investigar. Su mayor preocupación se centraba en la geometría y la aplicación del cálculo diferencial y la matemática sublime a la geometría; además de ser inventor de dos barómetros y del famoso comparador utilizado en la Oficina de Pesas y Medidas de París para determinar los patrones del metro. Su ciencia siempre estuvo al servicio de la ciudadanía, y de ahí que aceptara ofrecer sus conocimientos para servir a su país y a sus ciudadanos en nombre del gobierno español, como representante en la Oficina de pesas y Medidas de París.

Pero fue, además y por encima de todo, un ciudadano ilustrado amante de la libertad que supo defender a sus conciudadanos participando de los avances democráticos de las Cortes de Cádiz y firmando la solicitud de supresión de la Inquisición.

Por último, no queremos separar, para la total celebración de la memoria de un gran sabio y de la gloria de su genio, los hechos sociales y políticos que encumbran, más si cabe, su personalidad. ¿Cómo nos va a ser extraño que Pedrayes fuera defensor de la Ilustración científica y cultural, propia de los países más avanzados de su época?; ni que hubiera apoyado y promovido la disolución de la Inquisición, porque amaba la ciencia, pero también amaba la libertad.

Agustín de Pedrayes debe ser recordado por sus hallazgos sobre Cuadraturas y rectificación de curvas con aplicación del cálculo diferencial e integral; por su aportación al actual Sistema de Pesas y Medidas; por la creación de varios aparatos de medida; por la importante contribución a los programas de enseñanza de la matemática de su época. Y sobre todo por el legado de ejemplo de persona sencilla y dedicada al estudio de la ciencia a tiempo completo. Pedrayes es el recuerdo vivo de su infinita perseverancia y de su esfuerzo en la consecución de sus logros.

Sus amigos y contemporáneos así lo valoraron. El propio Jovellanos, cuando se enteró de que regresaba a Madrid en 1795, en contra de su opinión, tras la recuperación de su larga enfermedad, mostró su desacuerdo porque sabía el cerebro que perdía.

Se preciaba Pedrayes de su relación con científicos y matemáticos como Méchain o Delambre y con personalidades como Jovellanos, con los que mantuvo muchos puntos de colaboración.

Como decía Laplace en lecho de muerte: «Lo que conocemos es poco; lo que ignoramos es inmenso». Con el legado de Agustín de Pedrayes hemos cono- 
cido algo más e ignorado algo menos. Agustín de Pedrayes ensanchó el ámbito del pensamiento científico de su época y ayudó a la consecución de una mayor dignidad y una mayor libertad, revelando a nuestra vista la majestuosidad de la matemática. Los esfuerzos de cientos de sabios matemáticos a lo largo de muchos siglos, iluminaban con fulgente luz una de las ramas más excitante de la matemática.

Creo haber presentado así, la vida de un asturiano célebre, de una vida ilustre consagrada a la gloria de la matemática, en una época llena de insignes ilustrados. El nombre de Pedrayes honra a la región de Asturias, que junto a sus figuras más destacadas, son motivo de orgullo para todos los ciudadanos y deberían ser acicate para su eterno recuerdo y valoración. La voz de Asturias y de España entera, debería alzarse para reivindicar la memoria de tan ilustre científico. He querido celebrar aquí la memoria de un gran sabio de origen asturiano, poco estudiado por los científicos que desprecian los logros de otras épocas y olvidado por los historiadores, en general preocupados en otras historiografías. Un ilustrado que la ignorancia olvidó por mimetismo de lo que venga más allá de los Pirineos. Mas no podemos separar el autor de su obra y de su época. 